BUHEP-00-19

HUPT-01/A005

hep-ph/0012256

\title{
Composite Scalars at LEP: Constraining Technicolor Theories
}

\author{
Kevin R. Lynch*, 1, Elizabeth H. Simmons ${ }^{\dagger, 1,2}$ \\ ${ }^{1}$ Department of Physics, Boston University, \\ 590 Commonwealth Avenue, Boston MA 02215 \\ ${ }^{2}$ Radcliffe Institute for Advanced Study, Harvard University, \\ 34 Concord Avenue, Cambridge, MA 02138 \\ and \\ Jefferson Physical Laboratory, Harvard University \\ Cambridge, MA 02138
}

March 14, 2001

\begin{abstract}
LEP I and LEP II data can be used to constrain technicolor models with light, neutral pseudo-NambuGoldstone bosons, $\mathrm{P}^{\mathrm{a}}$. We use published limits on branching ratios and cross sections for final states with photons, large missing energy, jet pairs, or b $\bar{b}$ pairs to constrain the anomalous $\mathrm{P}^{\mathrm{a}} \mathrm{Z}^{0} \mathrm{Z}^{0}, \mathrm{P}^{\mathrm{a}} \mathrm{Z}^{0} \gamma$, and $\mathrm{P}^{\mathrm{a}} \gamma \gamma$ couplings. From these results, we derive bounds on the size of the technicolor gauge group and the number of technifermion doublets in models such as Low-scale Technicolor.
\end{abstract}

\footnotetext{
*krlynch@bu.edu

†simmons@bu.edu
} 


\section{Introduction}

Although the scale of electroweak symmetry breaking is well established, the mechanism of that breaking is still unknown. Data collected at LEP over the last twelve years, however, have provided many constraints on the properties of that mechanism. In this paper, we consider what the LEP data reveal about nonminimal technicolor models. In particular, we explore how the limits on rare processes constrain technicolor models with neutral pseudo-Nambu-Goldstone bosons (PNGBs), $\mathrm{P}^{\mathrm{a}}$, which couple, through an anomaly, to the neutral electroweak bosons. PNGBs lighter than the $\mathrm{Z}^{0}$ can be produced at the $\mathrm{Z}^{0}$ pole through the decays $\mathrm{Z}^{0} \rightarrow \gamma \mathrm{P}^{\mathrm{a}}$ or $\mathrm{Z}^{0} \rightarrow \mathrm{Z}^{*} \mathrm{P}^{\mathrm{a}}$, while heavier PNGBs can be produced through a number of processes at the higher energies found at LEP II. Depending on the details of the specific model, the final state following PNGB decay may include jets, photons, or missing energy, providing striking signatures.

Our analysis is not the first to consider these processes $[1,2,3,4,5]$. Since the work of Reference [3], however, the LEP collaborations have published new analyses using additional LEP I data [6, 7, 8, 9, 10], allowing stronger limits to be placed on the $\mathrm{P}^{\mathrm{a}} \mathrm{Z}^{0} \gamma$ couplings. Furthermore, improvements in the resolution of photon energy measurements allow the limits to be extended to larger PNGB masses. The quality of the final LEP I data are such that, contrary to previous expectations, bounds can even be placed on the $\mathrm{P}^{a} \mathrm{Z}^{0} \mathrm{Z}^{0}$ coupling. Finally, some of the data collected at LEP II has been analyzed $[11,12,13,14,15,16]$ and provides a means both to search for heavier PNGBs and to place bounds on the $\mathrm{P}^{\mathrm{a}} \gamma \gamma$ couplings for the first time. The constraints on modern, non-minimal technicolor models derived from all of these coupling bounds are phenomenologically interesting.

In the next section, we review the production and primary decay mechanisms for technicolor PNGBs at LEP through the anomalies. In Section 3, we analyze the limits on the anomalous $\mathrm{P}^{\mathrm{a}} \mathrm{Z}^{0} \gamma$ and $\mathrm{P}^{\mathrm{a}} \mathrm{Z}^{0} \mathrm{Z}^{0}$ couplings that can be derived from published analyses of LEP I data. In Section 4 we likewise derive bounds on the anomalous $\mathrm{P}^{\mathrm{a}} \gamma \gamma, \mathrm{P}^{\mathrm{a}} \mathrm{Z}^{0} \gamma$ and $\mathrm{P}^{\mathrm{a}} \mathrm{Z}^{0} \mathrm{Z}^{0}$ couplings from published analyses of LEP II data. Section 5 compiles the strongest results for each anomalous coupling and $\mathrm{P}^{\mathrm{a}}$ decay mode. In Section 6 we determine what these results imply for various technicolor scenarios. We present our conclusions and thoughts about the future in Section 7.

\section{Production and decay of $\mathrm{P}^{\mathrm{a}}$}

At LEP I, a light neutral PNGB, $\mathrm{P}^{\mathrm{a}}$, with $M_{\mathrm{P}^{\mathrm{a}}}<M_{\mathrm{Z}^{0}}$ is primarily produced $[1,2,3]$ through an anomalous coupling to the $\mathrm{Z}^{0}$ boson and either a photon $\left(\mathrm{Z}^{0} \rightarrow \gamma \mathrm{P}^{\mathrm{a}}\right)$ or a second, off-shell $\mathrm{Z}^{0}$ boson $\left(\mathrm{Z}^{0} \rightarrow \mathrm{Z}^{*} \mathrm{P}^{\mathrm{a}}\right)$. At the higher center of mass energies of LEP II, PNGBs over a wider range of masses can be produced through $s$-channel $\gamma^{*} / \mathrm{Z}^{*}$ exchange and through a $2 \rightarrow 3$ production mechanism [4, 5]. For reference, we provide Feynman diagrams in Figures 1 and 2.

The anomalous coupling between the PNGB and the gauge bosons $\mathrm{G}_{1}$ and $\mathrm{G}_{2}$ is given, in a model with technicolor group $\mathrm{SU}\left(N_{\mathrm{TC}}\right)$, by an expression analogous to that for the QCD pion $[17,18,19]$

$$
N_{\mathrm{TC}} \mathcal{A}_{G_{1} G_{2}} \frac{g_{1} g_{2}}{2 \pi^{2} f_{\mathrm{P}^{\mathrm{a}}}} \epsilon_{\mu \nu \lambda \sigma} k_{1}^{\mu} k_{2}^{\nu} \varepsilon_{1}^{\lambda} \varepsilon_{2}^{\sigma}
$$

where $N_{\mathrm{TC}}$ is the number of technicolors, $\mathcal{A}_{G_{1} G_{2}}$ is the anomaly factor (discussed further below), the $g_{i}$ are the gauge couplings of the gauge bosons, and the $k_{i}$ and $\varepsilon_{i}$ are the four-momenta and polarizations of the gauge bosons. The $\mathrm{P}^{\mathrm{a}}$ decay constant, $f_{\mathrm{P}}$, which corresponds to the QCD pion decay constant, $f_{\pi}$, is given by [2]

$$
f_{\mathrm{P}^{\mathrm{a}}}^{2}=\frac{v^{2}}{2 \operatorname{Tr}\left[\left(T_{L}-T_{R}\right)^{2}\right]}
$$




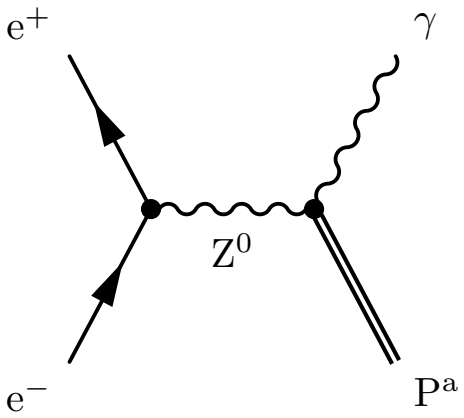

(a) $\mathrm{e}^{+} \mathrm{e}^{-} \rightarrow \mathrm{P}^{\mathrm{a}} \gamma$

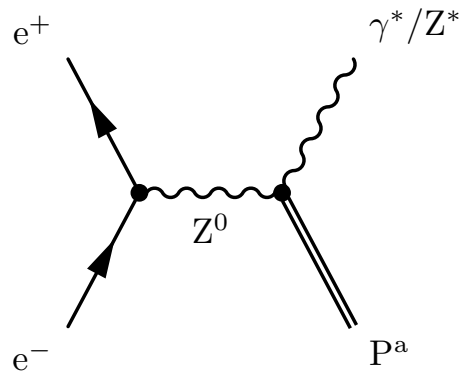

(b) $\mathrm{e}^{+} \mathrm{e}^{-} \rightarrow \mathrm{P}^{\mathrm{a}} \mathrm{Z}^{*}$

Figure 1: Primary production mechanisms of PNGBs at LEP I. At LEP I, processes with an intermediate, on-shell $\mathrm{Z}^{0}$ dominate the cross section for any PNGB production processes. The left hand diagram is the relevant one for processes with mono-energetic, hard photons, plus the $\mathrm{P}^{\mathrm{a}}$ decay products in the final state; these states give clean access to the $\mathrm{P}^{\mathrm{a}} \mathrm{Z}^{0} \gamma$ coupling. The right hand diagram is the relevant one for processes with four particles in the final state, and will generally give access to both the $\mathrm{P}^{\mathrm{a}} \mathrm{Z}^{0} \gamma$ and $\mathrm{P}^{\mathrm{a}} \mathrm{Z}^{0} \mathrm{Z}^{0}$ couplings.

where $v=246 \mathrm{GeV}$ is the weak scale, and $T_{L}\left(T_{R}\right)$ is the charged weak generator associated with the left-handed (right-handed) technifermions that comprise the PNGB. In the case of left-handed electroweak doublet techniquarks, Q (which are $\mathrm{SU}(3)_{C}$ triplets), and technileptons, $\mathrm{L}$ (which are $\mathrm{SU}(3)_{C}$ singlets), the above expression reduces to

$$
f_{\mathrm{Pa}}=\frac{v}{\sqrt{3 N_{\mathrm{Q}}+N_{\mathrm{L}}}}
$$

where the $N_{i}$ are the number of such electroweak doublets in the model. Equation 2.2 is only valid in the limit of small isospin breaking in the technifermion sector (in Section 6.1 we consider the consequences of a particular case of large isospin breaking).

The rate of PNGB production at the $\mathrm{Z}^{0}$ pole has previously been reported in the literature; the cross section for production at $\sqrt{s}=M_{\mathrm{Z}^{0}}$ is $[20]$

$$
\sigma\left(\mathrm{e}^{+} \mathrm{e}^{-} \rightarrow \mathrm{Z}^{0} \rightarrow \mathrm{P}^{\mathrm{a}} \mathrm{X}\right)=\sigma\left(\mathrm{e}^{+} \mathrm{e}^{-} \rightarrow \mathrm{Z}^{0}\right) \mathrm{BR}\left(\mathrm{Z}^{0} \rightarrow \mathrm{P}^{\mathrm{a}} \mathrm{X}\right)=\frac{12 \pi}{M_{\mathrm{Z}^{0}}^{2}} \mathrm{BR}\left(\mathrm{Z}^{0} \rightarrow \mathrm{e}^{+} \mathrm{e}^{-}\right) \mathrm{BR}\left(\mathrm{Z}^{0} \rightarrow \mathrm{P}^{\mathrm{a}} \mathrm{X}\right)
$$

Production in combination with a photon [1] has a width of

$$
\Gamma\left(\mathrm{Z}^{0} \rightarrow \gamma \mathrm{P}^{\mathrm{a}}\right)=2.3 \times 10^{-5} \mathrm{GeV}\left(\frac{123 \mathrm{GeV}}{f_{\mathrm{P}^{\mathrm{a}}}}\right)^{2}\left(1-\frac{M_{\mathrm{Pa}^{\mathrm{a}}}^{2}}{M_{\mathrm{Z}^{0}}^{2}}\right)^{3}\left(N_{\mathrm{TC}} \mathcal{A}_{\mathrm{Z}^{0} \gamma}\right)^{2}
$$

Since the measured $\mathrm{Z}^{0}$ width is $\Gamma_{\mathrm{Z}^{0}}=2.490 \mathrm{GeV}[20]$, we expect this branching ratio to be of order $10^{-5}$. The resulting final states contain a hard mono-energetic photon and the decay products of the $\mathrm{P}^{\mathrm{a}}$. Production in combination with an off-shell $\mathrm{Z}^{0}$ will be harder to observe. An upper bound on the decay width of the 


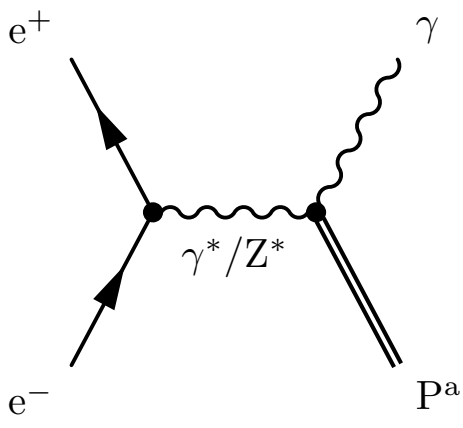

(a) $\mathrm{e}^{+} \mathrm{e}^{-} \rightarrow \mathrm{P}^{\mathrm{a}} \gamma$

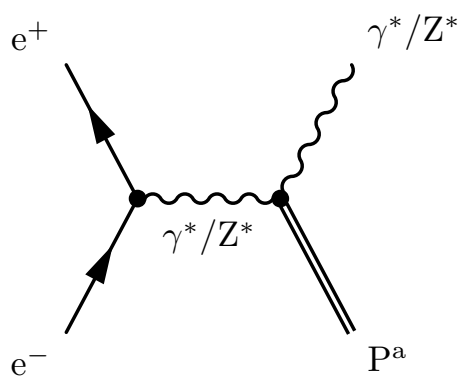

(c) $\mathrm{e}^{+} \mathrm{e}^{-} \rightarrow \mathrm{P}^{\mathrm{a}}\left(\gamma^{*} / \mathrm{Z}^{*}\right)$

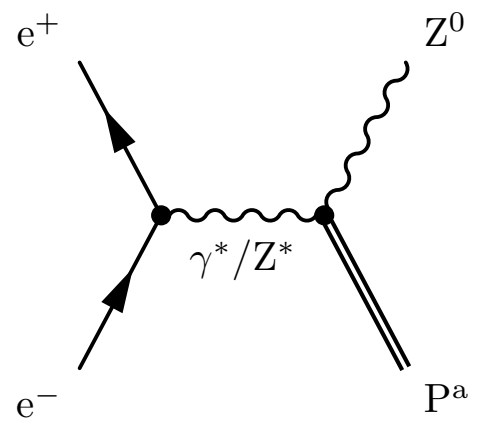

(b) $\mathrm{e}^{+} \mathrm{e}^{-} \rightarrow \mathrm{P}^{\mathrm{a}} \mathrm{Z}^{0}$

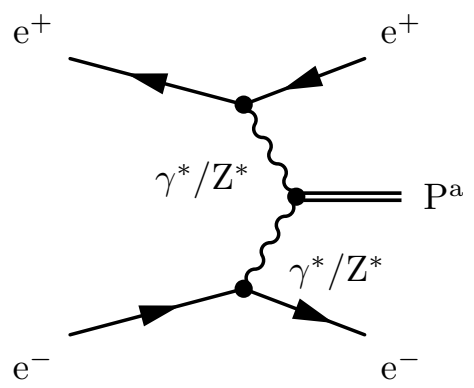

(d) $\mathrm{e}^{+} \mathrm{e}^{-} \rightarrow \mathrm{P}^{\mathrm{a}} \mathrm{e}^{+} \mathrm{e}^{-}$

Figure 2: Primary production mechanisms of PNGBs at LEP II. The first type of process is $s$-channel production via an intermediate, off-shell photon or $\mathrm{Z}^{0}$. The diagram at upper left is the relevant one for processes with a hard, mono-energetic photon plus the decay products of the $\mathrm{P}^{\mathrm{a}}$ in the final state, and gives access to both the $\mathrm{P}^{\mathrm{a}} \gamma \gamma$ and $\mathrm{P}^{\mathrm{a}} \mathrm{Z}^{0} \gamma$ couplings. The diagram at upper right is the relevant one for processes with a real $\mathrm{Z}^{0}$ plus the decay products of the $\mathrm{P}^{\mathrm{a}}$ in the final state, and gives access to both the $\mathrm{P}^{\mathrm{a}} \mathrm{Z}^{0} \gamma$ and $\mathrm{P}^{a} \mathrm{Z}^{0} \mathrm{Z}^{0}$ couplings. The diagram at lower left is also, in principle, of relevance at LEP II, and would give access to all of the various couplings of electroweak gauge bosons to PNGBs; however, these processes are much more difficult to analyze, and are not studied here. Finally, the diagram at lower right would, in principle, give access to all of the anomalous couplings of the $\mathrm{P}^{\mathrm{a}}$; however, kinematics strongly favors the process with intermediate photons, so that only the $\mathrm{P}^{\mathrm{a}} \gamma \gamma$ coupling is accessible. 
process $\mathrm{Z}^{0} \rightarrow \mathrm{Z}^{*} \mathrm{P}^{\mathrm{a}} \rightarrow \mathrm{P}^{\mathrm{a}} \mathrm{f} \overline{\mathrm{f}}$ is given in $[2] \mathrm{by}^{1}$

$$
\begin{gathered}
\Gamma\left(\mathrm{Z}^{0} \rightarrow \mathrm{P}^{\mathrm{a}} \mathrm{f} \overline{\mathrm{f}}\right)<7.6 \times 10^{-7} \mathrm{GeV}\left(\frac{123 \mathrm{GeV}}{f_{\mathrm{P}^{\mathrm{a}}}}\right)^{2} C_{f}\left(g_{L}^{2}+g_{R}^{2}\right)\left(N_{\mathrm{TC}} \mathcal{A}_{\mathrm{Z}^{0} \mathrm{Z}^{0}}\right)^{2}\left(\frac{M_{\mathrm{Z}^{0}}^{2}-M_{\mathrm{Pa}^{\mathrm{a}}}^{2}}{M_{\mathrm{Z}^{0}}^{6}}\right) \times \\
{\left[\left(M_{\mathrm{Z}^{0}}-M_{\mathrm{Pa}^{\mathrm{a}}}\right)^{2}\left(M_{\mathrm{Z}^{0}}^{2}-6 M_{\mathrm{Z}^{0}} M_{\mathrm{Pa}^{\mathrm{a}}}-5 M_{\mathrm{Pa}^{\mathrm{a}}}^{2}\right)-2 M_{\mathrm{P}^{\mathrm{a}}}^{2}\left(6 M_{\mathrm{Z}^{0}}^{2}-M_{\mathrm{P}^{\mathrm{a}}}^{2}\right) \log \left(\frac{2 M_{\mathrm{Z}^{0}} M_{\mathrm{Pa}^{\mathrm{a}}}-M_{\mathrm{Pa}^{\mathrm{a}}}^{2}}{M_{\mathrm{Z}^{0}}^{2}}\right)\right],}
\end{gathered}
$$

where $C_{f}$ is a color factor of 1 for leptons and 3 for quarks, and $g_{L}\left(g_{R}\right)$ is the left-handed (right-handed) coupling of the fermion $\mathrm{f}$ to the $\mathrm{Z}^{0}$. We expect branching ratios of order $10^{-7}$ to $10^{-6}$, depending on the process of interest.

The production cross section for a PNGB along with an electroweak gauge boson, $G$, at the higher center of mass energies of LEP II can be calculated, and has also been reported in the literature [2]. If $\sqrt{s}-M_{\mathrm{Pa}}>M_{G}$, it is possible to produce a PNGB in association with either an on-shell $\mathrm{Z}^{0}$ boson or a photon; the cross section well off the $\mathrm{Z}^{0}$ peak is given by

$$
\begin{aligned}
\sigma\left(\mathrm{e}^{+} \mathrm{e}^{-} \rightarrow \mathrm{P}^{\mathrm{a}} G\right)=\frac{\alpha_{\mathrm{em}}^{3} N_{\mathrm{TC}}^{2}}{6 \pi^{2} f_{\mathrm{Pa}}^{2} s} F(G) \lambda(s, & \left.M_{G}^{2}, M_{\mathrm{Pa}^{\mathrm{a}}}^{2}\right)^{3 / 2} \\
\times & {\left[\frac{\mathcal{A}_{\gamma G}^{2}}{s^{2}}+\frac{\mathcal{A}_{\gamma G} \mathcal{A}_{\mathrm{Z}^{0} G}\left(1-4 s_{W}^{2}\right)}{2 s_{W}^{2} c_{W}^{2} s\left(s-M_{\mathrm{Z}^{0}}^{2}\right)}+\frac{\mathcal{A}_{\mathrm{Z}^{0} G}^{2}\left(1-4 s_{W}^{2}+8 s_{W}^{4}\right)}{8 s_{W}^{4} c_{W}^{4}\left(s-M_{\mathrm{Z}^{0}}^{2}\right)^{2}}\right], }
\end{aligned}
$$

where $G$ is either the on-shell $\mathrm{Z}^{0}$ or $\gamma$ in the final state, $s_{W}^{4}=\sin ^{4} \theta_{W}, c_{W}^{4}=\cos ^{4} \theta_{W}, \lambda(a, b, c)=a^{2}+b^{2}+$ $c^{2}-2 a b-2 a c-2 b c$ and

$$
F(G)= \begin{cases}1 & G=\gamma \\ \frac{1}{s_{W}^{2} c_{W}^{2}} & G=\mathrm{Z}^{0} .\end{cases}
$$

In both cases, the first term is the photon exchange contribution, the third term is the $\mathrm{Z}^{0}$ exchange contribution, while the second term is the $\mathrm{Z}^{0} \gamma$ interference term (see Figure 2). Since $s_{W}^{2} \approx 0.23$, the interference contribution is generally negligible compared to the direct contributions.

The model-dependent value of the anomaly factor for the $\mathrm{P}^{\mathrm{a}} G_{1} G_{2}$ coupling which appears in those branching ratios is given by $[17,18,19]$

$$
4 \mathcal{A}_{G_{1} G_{2}}=\operatorname{Tr}\left[T^{a}\left(T_{1} T_{2}+T_{2} T_{1}\right)_{L}\right]+\operatorname{Tr}\left[T^{a}\left(T_{1} T_{2}+T_{2} T_{1}\right)_{R}\right],
$$

where $T^{a}$ is the generator of the axial current associated with $\mathrm{P}^{\mathrm{a}}$, the $T_{i}$ are the generators associated with the gauge boson $G_{i}$, and the subscripts $L$ and $R$ denote the left and right handed technifermion components that comprise $\mathrm{P}^{\mathrm{a}}$. The axial currents are defined as usual,

$$
j_{5}^{\mu a}=\bar{\psi} \gamma^{\mu} \gamma_{5} T^{a} \psi
$$

and the generators, $T^{a}$, are normalized such that

$$
\operatorname{Tr}\left(T^{a} T^{b}\right)=\frac{1}{2} \delta^{a b}
$$

\footnotetext{
${ }^{1}$ We have corrected here a slight error in the numerical coefficient of the formula as it appears in [2]. We have also included the color factor, $C_{f}$, which was omitted there.
} 
For the three cases with neutral electroweak gauge bosons, the anomaly factors are [1]

$$
\begin{gathered}
\mathcal{A}_{\gamma \gamma}=\operatorname{Tr}\left[T^{a} Q^{2}\right] \\
\mathcal{A}_{\mathrm{Z}^{0} \gamma}=\frac{1}{2} \operatorname{Tr}\left[T^{a}\left(T_{3 L}+T_{3 R}-2 Q \sin ^{2} \theta_{W}\right) Q\right] \\
\mathcal{A}_{\mathrm{Z}^{0} \mathrm{Z}^{0}}=\frac{1}{2} \operatorname{Tr}\left[T^{a}\left(\left(T_{3 L}-Q \sin ^{2} \theta_{W}\right)^{2}+\left(T_{3 R}-Q \sin ^{2} \theta_{W}\right)^{2}\right)\right] .
\end{gathered}
$$

We will explicitly evaluate the anomaly factors for a variety of models in Section 6 .

Our analyses will consider all of the dominant decay modes for the produced PNGBs. These fall into three classes:

1. In models where $\mathcal{A}_{\gamma \gamma} \neq 0$, the PNGB may decay through the anomaly to a pair of photons at a rate [2]

$$
\Gamma\left(\mathrm{P}^{\mathrm{a}} \rightarrow \gamma \gamma\right)=\left(\frac{N_{\mathrm{TC}} \mathcal{A}_{\gamma \gamma}}{f_{\mathrm{Pa}}}\right)^{2} \frac{\alpha_{\mathrm{em}}^{2}}{8 \pi^{3}} M_{\mathrm{P}^{\mathrm{a}}}^{3}
$$

Even for large $M_{\mathrm{Pa}}$, this decay width is very narrow; for example, with $M_{\mathrm{P}^{\mathrm{a}}}=M_{\mathrm{Z}^{0}}$ and $f_{\mathrm{P}^{\mathrm{a}}}=123 \mathrm{GeV}$, we find $\Gamma\left(\mathrm{P}^{\mathrm{a}} \rightarrow \gamma \gamma\right) \approx\left(N_{\mathrm{TC}} \mathcal{A}_{\gamma \gamma}\right)^{2} \times 10^{-1} \mathrm{keV}$.

2. The PNGB may decay invisibly into neutrinos or other long-lived neutral particles. Alternatively, the PNGB may be long-lived and escape the detector. In either case, $\mathrm{P}^{\mathrm{a}}$ will manifest as missing energy.

3. The PNGB may decay into hadrons. This may arise through decays into $q \bar{q}$ pairs, with $b \bar{b}$ being of particular interest in some models. Alternatively, PNGBs comprised of colored technifermions may decay into gluon pairs. If no flavor tagging is employed in the experimental analysis, limits on hadronic decays of the PNGB are assumed to apply equally well to quark and gluon decay modes.

Current experimental data provide bounds on all of these processes.

\section{Limits from LEP I}

In this section we explore the limits that can be obtained on the anomaly factors $\mathcal{A}_{\mathrm{Z}^{0} \gamma}$ and $\mathcal{A}_{\mathrm{Z}^{0} \mathrm{Z}^{0}}$ from published LEP I data $[6,7,8,9,10]$, collected at $\sqrt{s}=M_{\mathrm{Z}^{0}}$. We do so for a number of possible decay modes of the $\mathrm{P}^{\mathrm{a}}$. The relevant Feynman diagrams are displayed in Figure 1.

\subsection{Limits on $N_{\mathrm{TC}} \mathcal{A}_{\mathrm{Z}^{0} \gamma}$}

For a $\mathrm{Z}^{0}$ produced at rest and undergoing the two-body decay $\mathrm{Z}^{0} \rightarrow \gamma \mathrm{P}^{\mathrm{a}}$ (Figure 1a), energy-momentum conservation fixes the photon energy to be $[2]$

$$
E_{\gamma}=\frac{M_{\mathrm{Z}^{0}}^{2}-M_{\mathrm{P}^{\mathrm{a}}}^{2}}{2 M_{\mathrm{Z}^{0}}}
$$

This provides a striking set of signatures. We will now use LEP I data on final states that include at least one hard photon to derive limits on $N_{\mathrm{TC}} \mathcal{A}_{\mathrm{Z}^{0} \gamma}$. 


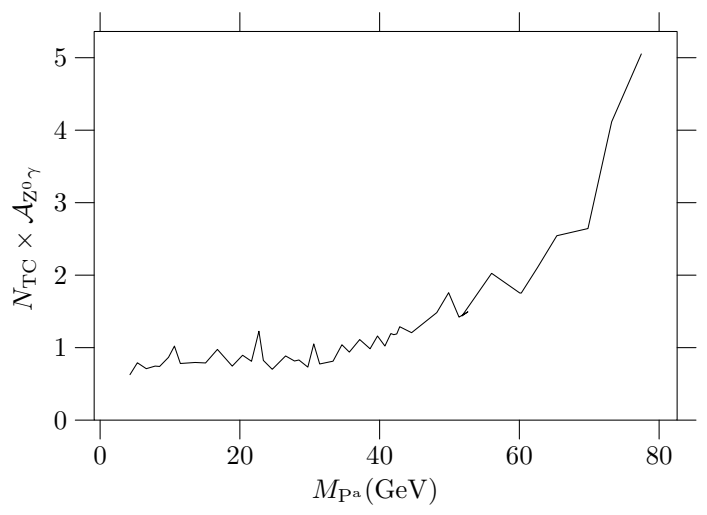

Figure 3: Upper limits at $95 \%$ c.l. on $N_{\mathrm{TC}} \mathcal{A}_{\mathrm{Z}^{0} \gamma}\left(123 \mathrm{GeV} / f_{\mathrm{P}^{\mathrm{a}}}\right)$ from the process $\mathrm{Z}^{0} \rightarrow \gamma \mathrm{P}^{\mathrm{a}} \rightarrow \gamma \gamma \gamma$. Our results are derived from an L3 analysis [6] assuming the PNGB has essentially zero width. Fluctuations in the curves arise from fluctuations in the data.

\subsection{1 $\quad \mathrm{Z}^{0} \rightarrow \gamma \mathrm{P}^{\mathrm{a}} \rightarrow \gamma \gamma \gamma$}

If the PNGB decays dominantly to photons, a final state with three hard photons results (Figure 1a with $\mathrm{P}^{\mathrm{a}} \rightarrow \gamma \gamma$ ). The L3 collaboration has published limits on the production of a narrow resonance, $\mathrm{X}$, decaying to photons, based on $65.8 \mathrm{pb}^{-1}$ of data collected on and near the $\mathrm{Z}^{0}$ pole [6]. They find no evidence for a new resonance, and place $95 \%$ c.l. upper limits on the branching ratio $\mathrm{BR}\left(\mathrm{Z}^{0} \rightarrow \gamma \mathrm{X}\right) \mathrm{BR}(\mathrm{X} \rightarrow \gamma \gamma)$ as a function of $M_{\mathrm{X}}$. For $3 \mathrm{GeV}<M_{\mathrm{X}}<89 \mathrm{GeV}$, they find $\mathrm{BR}\left(\mathrm{Z}^{0} \rightarrow \gamma \mathrm{X}\right) \mathrm{BR}(\mathrm{X} \rightarrow \gamma \gamma)<1.3 \times 10^{-5}$.

Using Equation 2.5, we translate these data into upper bounds on $N_{\mathrm{TC}} \mathcal{A}_{\mathrm{Z}^{0} \gamma}$. Assuming $\operatorname{BR}\left(\mathrm{P}^{\mathrm{a}} \rightarrow \gamma \gamma\right) \approx 1$ and $f_{\mathrm{Pa}}=123 \mathrm{GeV}$, we find $N_{\mathrm{TC}} \mathcal{A}_{\mathrm{Z}^{0} \gamma}<0.5-2$ for PNGB masses below $60 \mathrm{GeV}$. Above $60 \mathrm{GeV}$, the data become rapidly less constraining (see Figure 3). These limits are a factor of two stronger than those in Reference [3].

\subsection{2 $\quad \mathrm{Z}^{0} \rightarrow \gamma \mathrm{P}^{\mathrm{a}} \rightarrow \gamma \mathrm{F}$}

If the predominant decays of the PNGB are invisible, or if it escapes the detector before decaying, then we expect a final state with one hard photon and missing energy (Figure 1a with $\mathrm{P}^{\mathrm{a}} \rightarrow \mathrm{E}$ ). The DELPHI collaboration has searched for anomalous single photon events, in $67.6 \mathrm{pb}^{-1}$ of data collected on and near the $\mathrm{Z}^{0}$ pole [7]. They derive $95 \%$ c.l. upper limits on the production cross section, $\sigma_{\mathrm{X}}$, of a narrow $\left(\Gamma_{\mathrm{X}}<2 \mathrm{GeV}\right)$ invisible particle $\mathrm{X}$ produced in association with a single hard photon, with the photon in the angular range $|\cos \theta|<0.7$ relative to the beamline. For $M_{\mathrm{X}}<M_{\mathrm{Z}^{0}}$, DELPHI provides limits on $\sigma_{\mathrm{X}}$ as a function of $M_{\mathrm{X}}$; the upper limit never exceeds $0.1 \mathrm{pb}$.

Since the $\mathrm{Z}^{0}$ decay is isotropic, we can scale our predictions to reflect the DELPHI angular coverage. If we assume that $\mathrm{P}^{\mathrm{a}}$ is always invisible and $f_{\mathrm{Pa}}=123 \mathrm{GeV}$, then using Equation 2.5, we can derive limits on $\mathrm{BR}\left(\mathrm{Z}^{0} \rightarrow \gamma \mathrm{P}^{\mathrm{a}}\right)$, and, hence, $N_{\mathrm{TC}} \mathcal{A}_{\mathrm{Z}^{0} \gamma}$. We find $N_{\mathrm{TC}} \mathcal{A}_{\mathrm{Z}^{0} \gamma}<0.5-1.2$ for $\mathrm{P}^{\mathrm{a}}$ masses below $60 \mathrm{GeV}$; the limits weaken at higher masses. The limits we obtain here are stronger than those based on the OPAL [21] data in Reference [3] and cover a larger mass range than those based on the L3 [22] data in Reference [3]. In the mass range $40 \mathrm{GeV}<M_{\mathrm{Pa}}<75 \mathrm{GeV}$ where data from all three experiments exist, the data from L3 give the strongest bounds. We plot our results in Figure 4, along with those of Reference [3].

OPAL has also published more recent results on $\gamma$ events, based on $160 \mathrm{pb}^{-1}$ of data collected near the $\mathrm{Z}^{0}$ pole [10]. However, since they present this data as limits on the branching ratios of heavy neutralinos to light neutralinos and photons via $\mathrm{Z}^{0} \rightarrow \tilde{\chi}_{2}^{0} \tilde{\chi}_{1}^{0} \rightarrow \tilde{\chi}_{1}^{0} \tilde{\chi}_{1}^{0} \gamma$, we can not use their results to constrain $N_{\mathrm{TC}} \mathcal{A}_{\mathrm{Z}^{0} \gamma}$. 


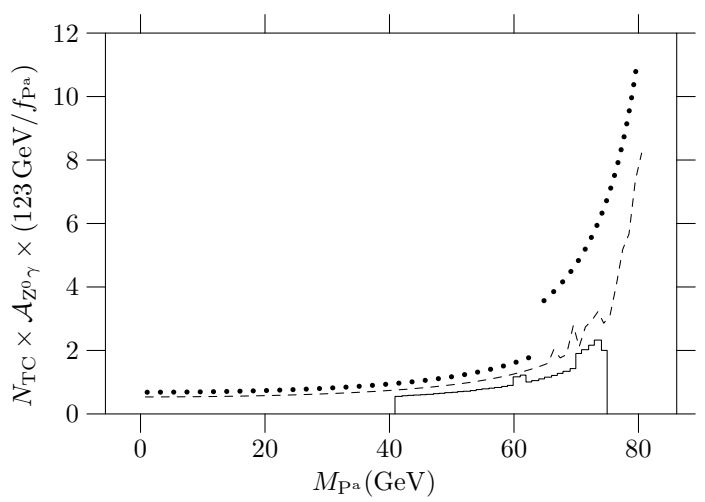

Figure 4: Upper limits at $95 \%$ c.l. on $N_{\mathrm{TC}} \mathcal{A}_{\mathrm{Z}^{0} \gamma}\left(123 \mathrm{GeV} / f_{\mathrm{P}^{\mathrm{a}}}\right)$ from the process $\mathrm{Z}^{0} \rightarrow \gamma \mathrm{P}^{\mathrm{a}} \rightarrow \gamma \mathrm{E}$. The dashed line corresponds to the results we derived from DELPHI data [7]. The dotted curves show the results derived from OPAL data [21] in Reference [3]; OPAL performed separate searches for scalars with masses below 80 and $60 \mathrm{GeV}$. The solid line shows limits extracted from L3 data [22] in Reference [3].

\subsection{3 $\quad \mathrm{Z}^{0} \rightarrow \gamma \mathrm{P}^{\mathrm{a}} \rightarrow \gamma$ jet jet}

If the dominant decay mode of the PNGB is hadronic, a final state with one hard photon and a pair of jets is expected (Figure 1a with $\mathrm{P}^{\mathrm{a}} \rightarrow$ jet jet). Both the OPAL and L3 collaborations have published limits on this process.

OPAL has searched for new, narrow particles decaying to hadrons with an associated hard photon in $140 \mathrm{pb}^{-1}$ of $\mathrm{Z}^{0}$ pole data [9]. They present two sets of relevant limits: a search for a scalar resonance, $\mathrm{S}^{0}$, which decays hadronically, and a search assuming that $\mathrm{S}^{0}$ decays predominantly to $\mathrm{b} \overline{\mathrm{b}}$. They find no evidence for production in either mode, and place $95 \%$ c.l. upper limits on the product of branching ratios, $\operatorname{BR}\left(Z^{0} \rightarrow \gamma \mathrm{S}^{0}\right) \mathrm{BR}\left(\mathrm{S}^{0} \rightarrow \mathrm{q} \overline{\mathrm{q}}\right)$ as a function of $M_{\mathrm{S}^{0}}$. For $20 \mathrm{GeV}<M_{\mathrm{S}^{0}}<80 \mathrm{GeV}$, the limit always satisfies $\operatorname{BR}\left(Z^{0} \rightarrow \gamma \mathrm{S}^{0}\right) \mathrm{BR}\left(\mathrm{S}^{0} \rightarrow \mathrm{q} \overline{\mathrm{q}}\right)<2 \times 10^{-5}$. Using Equation 2.5, we translate these limits into upper bounds on $N_{\mathrm{TC}} \mathcal{A}_{\mathrm{Z}^{0} \gamma}$, assuming that $f_{\mathrm{Pa}}=123 \mathrm{GeV}$. Both sets of data provide limits $N_{\mathrm{TC}} \mathcal{A}_{\mathrm{Z}^{0} \gamma}<1-3$ for PNGB masses below $60 \mathrm{GeV}$, and $N_{\mathrm{TC}} \mathcal{A}_{\mathrm{Z}^{0} \gamma}<10-15$ for PNGB masses below $80 \mathrm{GeV}$.

The L3 collaboration has also searched for new, narrow scalar particles, $\mathrm{H}^{0}$, decaying to hadrons with an associated hard photon in $96.8 \mathrm{pb}^{-1}$ of data collected at the $\mathrm{Z}^{0}$ pole [8]. They find no evidence for a new particle, and place $95 \%$ c.l. upper limits on the the cross section for the process $\mathrm{Z}^{0} \rightarrow \gamma \mathrm{H}^{0} \rightarrow \gamma q \overline{\mathrm{q}}$. For $20 \mathrm{GeV}<M_{\mathrm{H}^{0}}<80 \mathrm{GeV}$, they find $\sigma\left(\mathrm{e}^{+} \mathrm{e}^{-} \rightarrow \mathrm{H}^{0} \gamma\right) \mathrm{BR}\left(\mathrm{H}^{0} \rightarrow \mathrm{q} \overline{\mathrm{q}}\right)<1 \mathrm{pb}$. Using Equation 2.5 we translate their full $M_{\mathrm{H}^{0}}$-dependent limits into upper bounds on $N_{\mathrm{TC}} \mathcal{A}_{\mathrm{Z}^{0} \gamma}$. Assuming $\mathrm{BR}\left(\mathrm{P}^{\mathrm{a}} \rightarrow \mathrm{q} \overline{\mathrm{q}}\right) \approx 1$ and $f_{\mathrm{Pa}}=123 \mathrm{GeV}$, we find limits $N_{\mathrm{TC}} \mathcal{A}_{\mathrm{Z}^{0} \gamma}<1-3$ for PNGB masses below $60 \mathrm{GeV}$, and $N_{\mathrm{TC}} \mathcal{A}_{\mathrm{Z}^{0} \gamma}<15$ for PNGB masses below $80 \mathrm{GeV}$.

As Figure 5 illustrates, the several limits on $N_{\mathrm{TC}} \mathcal{A}_{\mathrm{Z}^{0} \gamma}$ for hadronically-decaying PNGB are similar. They improve on the bounds in Reference [3] by a factor of two to three.

\subsection{Limits on $N_{\mathrm{TC}} \mathcal{A}_{\mathrm{Z}^{0} \mathrm{Z}^{0}}$}

We next obtain limits on $N_{\mathrm{TC}} \mathcal{A}_{\mathrm{Z}^{0} \mathrm{Z}^{0}}$ from the LEP I data. The relevant decay paths we examine include $\mathrm{Z}^{0} \rightarrow \mathrm{Z}^{*} \mathrm{P}^{\mathrm{a}} \rightarrow \not \mathrm{Eq} \overline{\mathrm{q}}$ (where the $\mathrm{P}^{\mathrm{a}}$ can decay either hadronically or invisibly) and $\mathrm{Z}^{0} \rightarrow \mathrm{Z}^{*} \mathrm{P}^{\mathrm{a}} \rightarrow \gamma \gamma \mathrm{q} \overline{\mathrm{q}}$, so that final states with two jets will dominate (Figure 1b).

In principle, we must also consider the contribution of an off-shell photon to the q⿱亠 production processes (Figure 1b), which would give a limit on $\mathcal{A}_{\mathrm{Z}^{0} \gamma}$; however, these results are numerically much weaker than the 


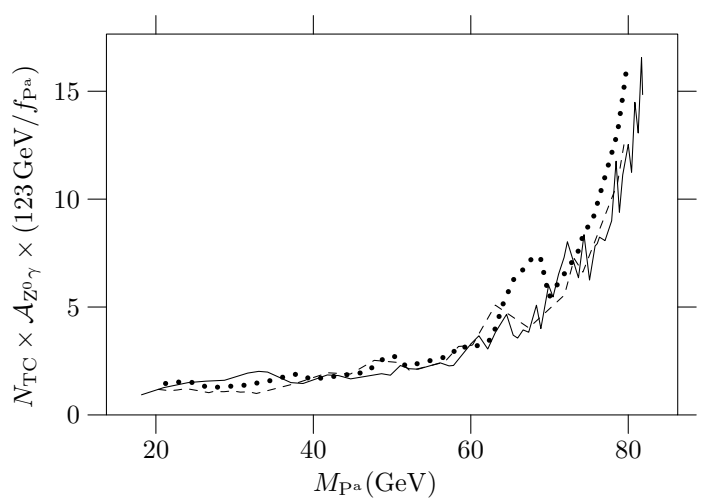

Figure 5: Upper limits at $95 \%$ c.l. on $N_{\mathrm{TC}} \mathcal{A}_{\mathrm{Z}^{0} \gamma}\left(123 \mathrm{GeV} / f_{\mathrm{P}^{\mathrm{a}}}\right)$ from $\mathrm{Z}^{0} \rightarrow \gamma \mathrm{P}^{\mathrm{a}} \rightarrow \gamma \mathrm{q} \overline{\mathrm{q}}$. We derived the dotted (dashed) curve from an OPAL [9] bound that assumes the new scalar decays to $\mathrm{q} \overline{\mathrm{q}}(\mathrm{b} \overline{\mathrm{b}})$. The solid curve comes from L3 [8] limits for scalar decays to hadrons. Fluctuations in the curves arise from fluctuations in the data.

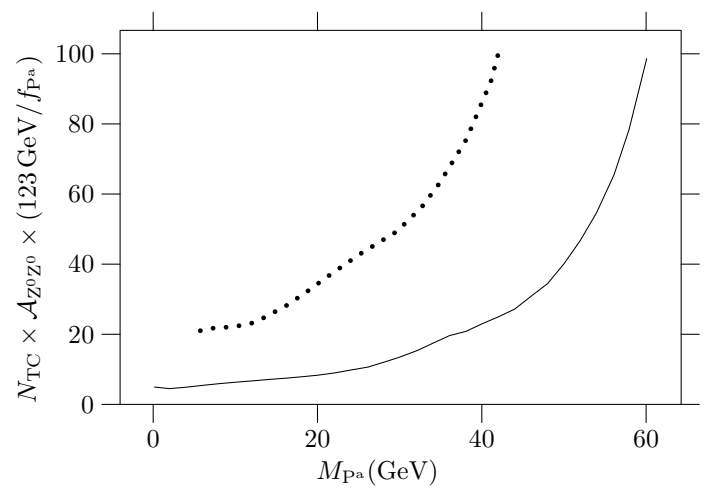

Figure 6: Upper limits at $95 \%$ c.l. on $N_{\mathrm{TC}} \mathcal{A}_{\mathrm{Z}^{0} \mathrm{Z}^{0}}\left(123 \mathrm{GeV} / f_{\mathrm{P}^{a}}\right)$ from $\mathrm{Z}^{0} \rightarrow \mathrm{Z}^{*} \mathrm{P}^{\mathrm{a}} \rightarrow \mathrm{q} \overline{\mathrm{q}} \mathrm{E}$, based on OPAL data [10]. The dotted curve denotes the limits on a hadronically decaying $\mathrm{P}^{\mathrm{a}}$, while the solid curve holds for an invisibly decaying $\mathrm{P}^{\mathrm{a}}$.

equivalent limits we obtained in Section 3.1. Therefore, we shall apply these limits on $N_{\mathrm{TC}} \mathcal{A}_{\mathrm{Z}^{0} \mathrm{Z}^{0}}$ only to models where $\mathcal{A}_{\mathrm{Z}^{0} \gamma} \ll \mathcal{A}_{\mathrm{Z}^{0} \mathrm{Z}^{0}}$, such as the Appelquist-Terning one-family model [23] discussed in Section 6.1.

\subsection{1 $\quad \mathrm{Z}^{0} \rightarrow \mathrm{Z}^{*} \mathrm{P}^{\mathrm{a}} \rightarrow$ jet jet $\mathrm{E}$}

This final state can arise in two ways: with the off-shell $\mathrm{Z}^{0}$ decaying hadronically and the PNGB decaying invisibly, or with the off-shell $Z^{0}$ decaying invisibly (to neutrino pairs) and the PNGB decaying hadronically (Figure 1b). The OPAL collaboration has searched for production of a scalar particle, $\mathrm{S}^{0}$, in both modes, based on $160 \mathrm{pb}^{-1}$ of data collected near the $\mathrm{Z}^{0}$ pole [10]. They find no evidence for either mode, and place $95 \%$ c.l. upper limits on the production cross section for $\mathrm{q} \overline{\mathrm{q}} \mathrm{E}$ through the intermediate state, $\mathrm{Z}^{*} \mathrm{~S}^{0}$, 


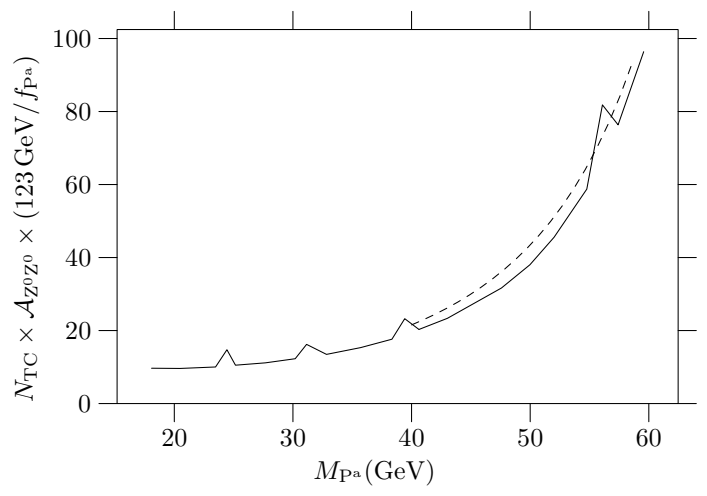

Figure 7 : Upper limits at $95 \%$ c.l. on $N_{\mathrm{TC}} \mathcal{A}_{\mathrm{Z}^{0} \mathrm{Z}^{0}}\left(123 \mathrm{GeV} / f_{\mathrm{P}^{\mathrm{a}}}\right)$ from the process $\mathrm{Z}^{0} \rightarrow \mathrm{Z}^{*} \mathrm{P}^{\mathrm{a}} \rightarrow \mathrm{q} \overline{\mathrm{q}} \gamma \gamma$. Our limits are based on L3 [8] and OPAL [9] data. The solid curve comes from L3 data, while the dashed curve comes from the OPAL data. Fluctuations in the curves arise from fluctuations in the data.

normalized to the production cross section for the Standard Model (SM) Higgs $\mathrm{Z}^{*} \mathrm{H}^{0}$ intermediate state ${ }^{2}$, $\sigma\left(\mathrm{e}^{+} \mathrm{e}^{-} \rightarrow \mathrm{H}_{\mathrm{SM}}^{0} \mathrm{Z}^{*}\right)$. We call their ratio of cross sections $R$. For the visible decay of the scalar, the numerator of $R$ is $\sigma\left(\mathrm{e}^{+} \mathrm{e}^{-} \rightarrow \mathrm{S}^{0} \mathrm{Z}^{*}\right) \mathrm{BR}\left(\mathrm{S}^{0} \rightarrow \mathrm{q} \overline{\mathrm{q}}\right)$, and we label the ratio $R_{\text {visible. For }} M_{\mathrm{S}^{0}}=5 \mathrm{GeV}$, the upper limit on $R_{\mathrm{visible}}$ is $10^{-3}$; this weakens to $R_{\mathrm{visible}} \leq 1$ as $M_{\mathrm{S}^{0}}$ increases to $65 \mathrm{GeV}$. For the invisible decay of the scalar, the numerator of $R$ is taken to be $\sigma\left(\mathrm{e}^{+} \mathrm{e}^{-} \rightarrow \mathrm{S}^{0} \mathrm{Z}^{*}\right)$, and we label the ratio $R_{\text {invisible. The upper limit on }}$ $R_{\text {invisible }}$ is $10^{-4}$ at $M_{\mathrm{S}^{0}}=0 \mathrm{GeV}$; this weakens to $R_{\mathrm{visible}} \leq 1$ as $M_{\mathrm{S}^{0}}$ rises toward $M_{\mathrm{Z}^{0}}$.

Using Equation 2.6, we derive upper bounds on $N_{\mathrm{TC}} \mathcal{A}_{\mathrm{Z}^{0} \mathrm{Z}^{0}}$. For a PNGB that (nearly) always decays to q $\bar{q}$ with $f_{\mathrm{Pa}}=123 \mathrm{GeV}$, we find $N_{\mathrm{TC}} \mathcal{A}_{\mathrm{Z}^{0} \mathrm{Z}^{0}}<20-50$ for PNGB masses below $30 \mathrm{GeV}$. For an invisibly decaying PNGB, we find $N_{\mathrm{TC}} \mathcal{A}_{\mathrm{Z}^{0} \mathrm{Z}^{0}}<5-13$ for PNGB masses below $30 \mathrm{GeV}$. In both cases, above $30 \mathrm{GeV}$, the data become rapidly less constraining. Our results appear in Figure 6 .

\subsection{2 $\mathrm{Z}^{0} \rightarrow \mathrm{Z}^{*} \mathrm{P}^{\mathrm{a}} \rightarrow$ jet jet $\gamma \gamma$}

If the PNGB decays predominantly to photons, a final state with two hard photons and two jets results (Figure $1 \mathrm{~b}$ with $\mathrm{P}^{\mathrm{a}} \rightarrow \gamma \gamma$ and $\mathrm{Z}^{*} \rightarrow \mathrm{q} \overline{\mathrm{q}}$ ). Both the L3 and OPAL collaborations have studied this final state.

L3 has published limits on the production of a scalar particle, $\mathrm{H}^{0}$, decaying to two photons and accompanied by hadrons, based on $96.8 \mathrm{pb}^{-1}$ of data collected near the $\mathrm{Z}^{0}$ pole [8]. They find no evidence for this mode, and place $95 \%$ c.l. upper limits on the production cross section as a function of $M_{\mathrm{H}^{0}}$. For $20 \mathrm{GeV}<M_{\mathrm{H}^{0}}<70 \mathrm{GeV}$, the collaboration finds $\sigma\left(\mathrm{e}^{+} \mathrm{e}^{-} \rightarrow \mathrm{H}^{0}+\right.$ hadrons) $\mathrm{BR}\left(\mathrm{H}^{0} \rightarrow \gamma \gamma\right)<10^{-1} \mathrm{pb}$.

The OPAL collaboration has also published limits on the production of a photonically decaying scalar, $\mathrm{S}^{0}$, in this mode, based on $140 \mathrm{pb}^{-1}$ of data collected on and near the $\mathrm{Z}^{0}$ pole [9]. They find no evidence for this mode. For particle masses in the range $40 \mathrm{GeV}<M_{\mathrm{S}^{0}}<80 \mathrm{GeV}$, OPAL finds a $95 \%$ c.l. limit on the

$$
\begin{aligned}
& { }^{2} \text { The SM Higgs branching ratio can be found in the literature }[24,25] \\
& \begin{array}{r}
\frac{\mathrm{BR}\left(\mathrm{Z}^{0} \rightarrow \mathrm{H}^{0} \mathrm{f} \overline{\mathrm{f}}\right)}{\mathrm{BR}\left(\mathrm{Z}^{0} \rightarrow \mathrm{f} \overline{\mathrm{f}}\right)}=\frac{g^{2}}{192 \pi^{2} \cos ^{2} \theta_{W}}\left[\frac{3 y\left(y^{4}-8 y^{2}+20\right)}{\sqrt{4-y^{2}}} \cos ^{-1}\left(\frac{y\left(3-y^{2}\right)}{2}\right)-\right. \\
\left.3\left(y^{4}-6 y^{2}+4\right) \ln y-\frac{1}{2}\left(1-y^{2}\right)\left(2 y^{4}-13 y^{2}+47\right)\right]
\end{array}
\end{aligned}
$$

where $y=M_{\mathrm{H}^{0}} / M_{\mathrm{Z}^{0}}>\Gamma_{\mathrm{Z}^{0}} / M_{\mathrm{Z}^{0}}$. This approximation neglects the masses of the fermions $\mathrm{f}$, and the $\mathrm{Z}^{0}$ width, $\Gamma_{\mathrm{Z}^{0}}$, which is acceptable for $y>\Gamma_{Z^{0}} / M_{Z^{0}}$. Using this branching ratio, we can derive the necessary cross section. 
product of branching ratios, $\mathrm{BR}\left(\mathrm{Z}^{0} \rightarrow \mathrm{S}^{0} \mathrm{q} \overline{\mathrm{q}}\right) \mathrm{BR}\left(\mathrm{S}^{0} \rightarrow \gamma \gamma\right)<2 \times 10^{-6}$. For smaller masses, $M_{\mathrm{S}^{0}}<40 \mathrm{GeV}$, OPAL states that the limits are weaker, but does not provide numerical values.

Using Equation 2.6, we infer upper bounds on $N_{\mathrm{TC}} \mathcal{A}_{\mathrm{Z}^{0} \mathrm{Z}^{\mathrm{O}}}$ in models with PNGB decays dominated by two photon states and $f_{\mathrm{P}^{\mathrm{a}}}=123 \mathrm{GeV}$. For PNGB masses below $30 \mathrm{GeV}$, we find limits $N_{\mathrm{TC}} \mathcal{A}_{\mathrm{Z}^{0} \mathrm{Z}^{0}}<10-12$ from the L3 results. In the higher mass range where the L3 and OPAL data overlap, they provide nearly identical upper limits on $N_{\mathrm{TC}} \mathcal{A}_{\mathrm{Z}^{0} \mathrm{Z}^{0}}$ which become weaker with increasing $\mathrm{P}^{\mathrm{a}}$ mass, as shown in Figure 7.

\section{Limits from LEP II}

In this section we explore the limits that can be obtained on the anomaly factors $\mathcal{A}_{\gamma \gamma}, \mathcal{A}_{\mathrm{Z}^{0} \gamma}$, and $\mathcal{A}_{\mathrm{Z}^{0} \mathrm{Z}^{0}}$ from published LEP II data collected at energies well above the $\mathrm{Z}^{0}$ pole $[11,12,13,14,15,16]$. We do so for a number of possible decay modes of the $\mathrm{P}^{\mathrm{a}}$; the $\mathrm{P}^{\mathrm{a}}$ decay products will be accompanied either by a hard photon, the decay products of an on-shell $\mathrm{Z}^{0}$, or an $\mathrm{e}^{+} \mathrm{e}^{-}$pair. In all of the cases that we analyze below, the final state can arise through either an $s$-channel virtual photon or $\mathrm{Z}^{0}$, or a $2 \rightarrow 3$ body process. The Feynman diagrams for these processes are displayed in Figure 2. From Equation 2.7, we see that all final states will thus provide a simultaneous limit, either on $\mathcal{A}_{\mathrm{Z}^{0} \gamma}$ and $\mathcal{A}_{\gamma \gamma}$ (for a final state photon), or on $\mathcal{A}_{\mathrm{Z}^{0} \mathrm{Z}^{0}}$ and $\mathcal{A}_{\mathrm{Z}^{0} \gamma}$ (for a final state, on-shell $\mathrm{Z}^{0}$ ). In all cases, in order to separate these effects, we first note that the interference term in Equation 2.7 is negligible. In addition, we assume that one or the other of the direct terms dominates; that assumption is valid in all of the explicit models we examine in Section 6 . From Equation 2.7, we define the cross sections for processes with a final state photon by

$$
\sigma_{\gamma \gamma}^{\gamma}=\frac{\alpha_{\mathrm{em}}^{3}\left(s-M_{\mathrm{Pa}}^{2}\right)^{3}}{6 \pi^{2} f_{\mathrm{Pa}}^{2} s^{3}}\left(N_{\mathrm{TC}} \mathcal{A}_{\gamma \gamma}\right)^{2},
$$

for photon-dominated intermediate states, and

$$
\sigma_{\mathrm{Z}^{0} \gamma}^{\gamma}=\frac{\alpha_{\mathrm{em}}^{3}\left(1-4 s_{W}^{2}+8 s_{W}^{4}\right)\left(s-M_{\mathrm{Pa}}^{2}\right)^{3}}{48 \pi^{2} f_{\mathrm{Pa}}^{2} s_{W}^{4} c_{W}^{4} s\left(s-M_{\mathrm{Z}^{0}}^{2}\right)^{2}}\left(N_{\mathrm{TC}} \mathcal{A}_{\mathrm{Z}^{0} \gamma}\right)^{2},
$$

for $\mathrm{Z}^{0}$-dominated intermediate states. We similarly define the cross sections for processes with a final state $\mathrm{Z}^{0}$ by

$$
\sigma_{\mathrm{Z}^{0} \gamma}^{\mathrm{Z}^{0}}=\frac{\alpha_{\mathrm{em}}^{3} \lambda\left(s, M_{\mathrm{Z}^{0}}^{2}, M_{\mathrm{Pa}^{\mathrm{a}}}^{2}\right)^{3 / 2}}{6 \pi^{2} f_{\mathrm{P}^{\mathrm{a}}}^{2} s_{W}^{2} c_{W}^{2} s^{3}}\left(N_{\mathrm{TC}} \mathcal{A}_{\mathrm{Z}^{0} \gamma}\right)^{2}
$$

for photon-dominated intermediate states, and

$$
\sigma_{\mathrm{Z}^{0} \mathrm{Z}^{0}}^{\mathrm{Z}^{0}}=\frac{\alpha_{\mathrm{em}}^{3} \lambda\left(s, M_{\mathrm{Z}^{0}}^{2}, M_{\mathrm{Pa}^{\mathrm{a}}}^{2}\right)^{3 / 2}}{48 \pi^{2} f_{\mathrm{Pa}}^{2} s_{W}^{6} c_{W}^{6} s\left(s-M_{\mathrm{Z}^{0}}^{2}\right)^{2}}\left(N_{\mathrm{TC}} \mathcal{A}_{\mathrm{Z}^{0} \mathrm{Z}^{0}}\right)^{2}
$$

for $\mathrm{Z}^{0}$-dominated intermediate states. The function $\lambda(a, b, c)$ is given in Section 2 .

In this approximation, the limits set on $\mathcal{A}_{\gamma \gamma}$ and $\mathcal{A}_{Z^{0} \gamma}$ by processes with a final-state photon are related, because the same data is being used to separately constrain $\sigma_{\gamma \gamma}^{\gamma}$ and $\sigma_{\mathrm{Z}^{0} \gamma}^{\gamma}$. The limits that processes with a final-state $\mathrm{Z}^{0}$ set on $\mathcal{A}_{\mathrm{Z}^{0} \gamma}$ and $\mathcal{A}_{\mathrm{Z}^{0} \mathrm{Z}^{0}}$ are, likewise, related. By comparing the sizes of the factors preceding $\left(N_{\mathrm{TC}} \mathcal{A}_{G_{1} G_{2}}\right)^{2}$ in equations 4.1 and 4.2 (4.3 and 4.4), one may see that a LEP II limit on $N_{\mathrm{TC}} \mathcal{A}_{\mathrm{Z}^{0} \gamma}$ $\left(N_{\mathrm{TC}} \mathcal{A}_{\mathrm{Z}^{0} \mathrm{Z}^{0}}\right)$ is always stronger than the related limit on $N_{\mathrm{TC}} \mathcal{A}_{\gamma \gamma}\left(N_{\mathrm{TC}} \mathcal{A}_{\mathrm{Z}^{0} \gamma}\right)$, for any PNGB mass. In any specific model where the values of the anomaly factors are known, we can recombine ${ }^{3}$ the pair of implied limits on $N_{\mathrm{TC}}$ from $\sigma_{\gamma \gamma}^{\gamma}$ and $\sigma_{\mathrm{Z}^{0} \gamma}^{\gamma}$ (or from $\sigma_{\mathrm{Z}^{0} \gamma}^{\mathrm{Z}}$ and $\sigma_{\mathrm{Z}^{0} \mathrm{Z}^{0}}^{\mathrm{Z}^{0}}$ ) to obtain a single limit on $N_{\mathrm{TC}}$. We will not need to do this in the models discussed in Section 6 , as one of the paired anomaly factors always dominates.

\footnotetext{
${ }^{3}$ For example, take a process with a final-state photon and write the theoretical cross section as

$$
\sigma=\mathcal{F}_{\gamma \gamma}^{\gamma}\left(N_{\mathrm{TC}} \mathcal{A}_{\gamma \gamma}\right)^{2}+\mathcal{F}_{\mathrm{Z}^{0} \gamma}^{\gamma}\left(N_{\mathrm{TC}} \mathcal{A}_{\mathrm{Z}^{0} \gamma}\right)^{2}
$$
}



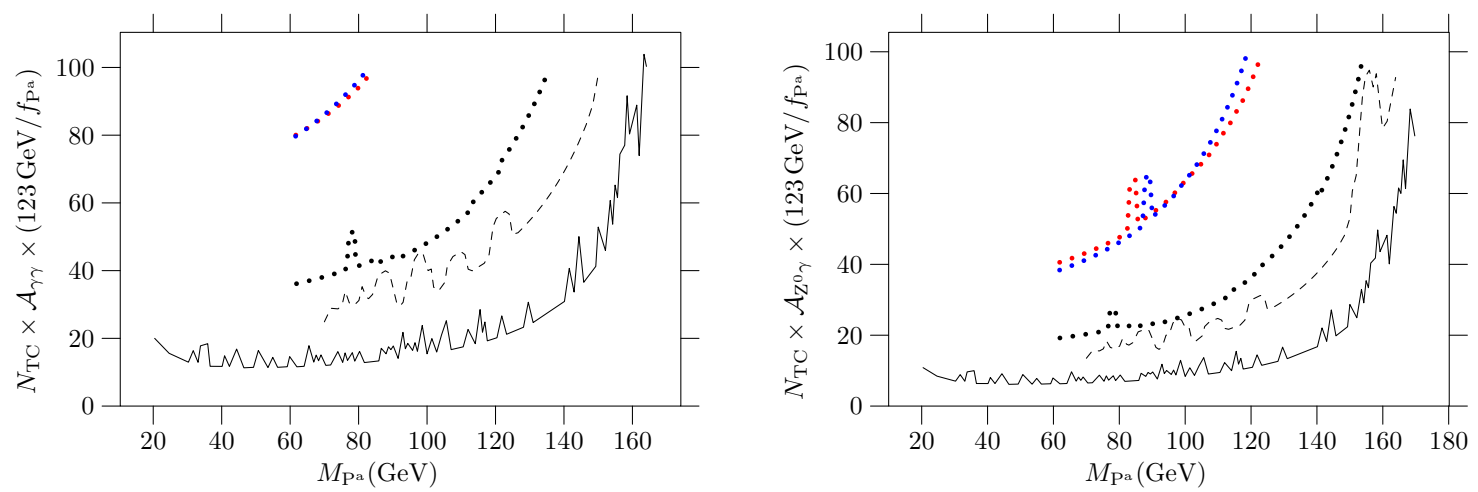

Figure 8: Upper limits at $95 \%$ c.l. on $N_{\mathrm{TC}} \mathcal{A}_{\gamma \gamma}\left(123 \mathrm{GeV} / f_{\mathrm{P}^{a}}\right)$ (at left) and $N_{\mathrm{TC}} \mathcal{A}_{\mathrm{Z}^{0} \gamma}\left(123 \mathrm{GeV} / f_{\mathrm{P}^{\mathrm{a}}}\right)$ (at right) from $\mathrm{e}^{+} \mathrm{e}^{-} \rightarrow \gamma \mathrm{P}^{\mathrm{a}} \rightarrow \gamma \gamma \gamma$. The solid line is derived from OPAL data [11]; the dashed line is derived from L3 data [12]; the three dotted lines come from DELPHI data [12] at various center of mass energies (from top to bottom, $161 \mathrm{GeV}, 172 \mathrm{GeV}$, and $183 \mathrm{GeV}$ ). Fluctuations in the curves arise from fluctuations in the data.

\subsection{Processes constraining both $N_{\mathrm{TC}} \mathcal{A}_{\gamma \gamma}$ and $N_{\mathrm{TC}} \mathcal{A}_{\mathrm{Z}^{0} \gamma}$}

\subsubsection{Limits from $\mathrm{e}^{+} \mathrm{e}^{-} \rightarrow \gamma \mathrm{P}^{\mathrm{a}} \rightarrow \gamma \gamma \gamma$}

If the PNGB decays predominantly to photons, the final state can contain three hard photons (Figure 2a with $\mathrm{P}^{\mathrm{a}} \rightarrow \gamma \gamma$ ). The DELPHI, L3, and OPAL collaborations have all published limits on this final state from their LEP II data samples.

The DELPHI collaboration has published limits on the production of a scalar resonance, $\mathrm{H}$, decaying to photons. They have performed three analyses, based on $9.7 \mathrm{pb}^{-1}$ of data collected at $\sqrt{s}=161 \mathrm{GeV}$, $10.1 \mathrm{pb}^{-1}$ of data collected at $\sqrt{s}=172 \mathrm{GeV}$, and $47.7 \mathrm{pb}^{-1}$ of data collected at $\sqrt{s}=183 \mathrm{GeV}[12]$. They find no evidence for a new resonance, and place $95 \%$ c.l. upper limits on the cross section $\sigma\left(\mathrm{e}^{+} \mathrm{e}^{-} \rightarrow \mathrm{H} \gamma\right) \mathrm{BR}(\mathrm{H} \rightarrow \gamma \gamma)$, as a function of $M_{\mathrm{H}}$. From data taken at $\sqrt{s}=183 \mathrm{GeV}$, they find $\sigma\left(\mathrm{e}^{+} \mathrm{e}^{-} \rightarrow \mathrm{H} \gamma\right) \mathrm{BR}(\mathrm{H} \rightarrow \gamma \gamma)<0.20 \mathrm{pb}$ within the mass range $60 \mathrm{GeV}<M_{\mathrm{H}}<184 \mathrm{GeV}$, almost independent of $M_{\mathrm{H}}$. The data taken at lower energies is less constraining (see Figure 8).

The L3 collaboration has published limits on the production of a scalar resonance, $\mathrm{H}$, decaying to photons. They have performed an analysis based on $176 \mathrm{pb}^{-1}$ of data collected at $\sqrt{s}=189 \mathrm{GeV}$ [13]. They find no evidence for a new resonance, and place $95 \%$ c.l. upper limits on the cross section $\sigma\left(\mathrm{e}^{+} \mathrm{e}^{-} \rightarrow \mathrm{H} \gamma\right) \mathrm{BR}(\mathrm{H} \rightarrow \gamma \gamma)$, as a function of $M_{\mathrm{H}}$. For $70 \mathrm{GeV}<M_{\mathrm{H}}<170 \mathrm{GeV}$, they find $\sigma\left(\mathrm{e}^{+} \mathrm{e}^{-} \rightarrow \mathrm{H} \gamma\right) \mathrm{BR}(\mathrm{H} \rightarrow \gamma \gamma)<0.30 \mathrm{pb}$, almost independent of $M_{\mathrm{H}}$.

The OPAL collaboration has also published limits on the production of a resonance, $\mathrm{X}$, decaying to photons. They have performed an analysis based on $178 \mathrm{pb}^{-1}$ of data collected at $\sqrt{s}=189 \mathrm{GeV}$ [11]. They find no evidence for a new resonance, and place $95 \%$ c.l. upper limits on the cross section $\sigma\left(\mathrm{e}^{+} \mathrm{e}^{-} \rightarrow \mathrm{X} \gamma\right) \mathrm{BR}(\mathrm{X} \rightarrow \gamma \gamma)$, as a function of $M_{\mathrm{X}}$. For $50 \mathrm{GeV}<M_{\mathrm{X}}<150 \mathrm{GeV}$, they find $\sigma\left(\mathrm{e}^{+} \mathrm{e}^{-} \rightarrow \mathrm{X} \gamma\right) \mathrm{BR}(\mathrm{X} \rightarrow \gamma \gamma)<0.03 \mathrm{pb}$, roughly independent of $M_{\mathrm{X}}$; for masses on either end of this range, their cross section limit becomes rapidly less

Then, if the experimental limit $\sigma \leq \sigma_{\text {data }}$ is taken to imply that $N_{\mathrm{TC}}^{\gamma}=\sqrt{\sigma_{\text {data }} / \mathcal{F}_{\gamma \gamma}^{\gamma} \mathcal{A}_{\gamma \gamma}^{2}}$ when photon-exchange dominates, a little manipulation shows that the more general limit is

$$
N_{\mathrm{TC}} \leq N_{\mathrm{TC}}^{\gamma}\left(1+\left(\frac{\mathcal{A}_{\mathrm{Z}^{0} \gamma}}{\mathcal{A}_{\gamma \gamma}}\right)^{2} \frac{\mathcal{F}_{\mathrm{Z}^{0} \gamma}^{\gamma}}{\mathcal{F}_{\gamma \gamma}^{\gamma}}\right)^{-\frac{1}{2}} .
$$



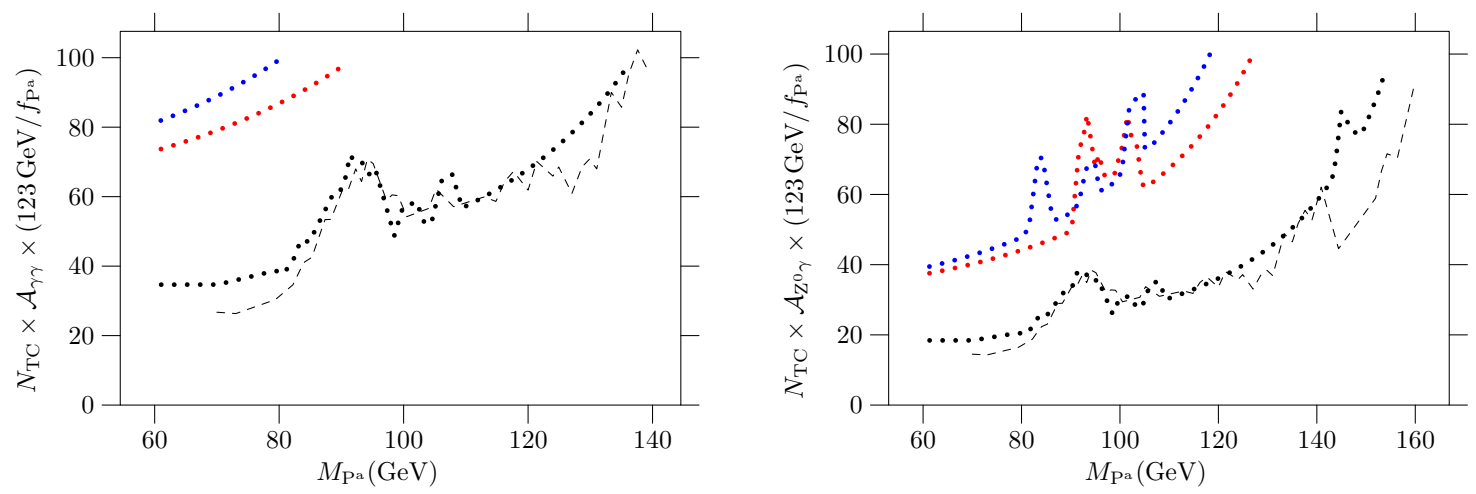

Figure 9: Upper limits at $95 \%$ c.l. on $N_{\mathrm{TC}} \mathcal{A}_{\gamma \gamma}\left(123 \mathrm{GeV} / f_{\mathrm{P}^{a}}\right)$ (at left) and $N_{\mathrm{TC}} \mathcal{A}_{\mathrm{Z}^{0} \gamma}\left(123 \mathrm{GeV} / f_{\mathrm{P}^{\mathrm{a}}}\right)$ (at right) from $\mathrm{e}^{+} \mathrm{e}^{-} \rightarrow \gamma \mathrm{P}^{\mathrm{a}} \rightarrow \gamma \mathrm{b} \overline{\mathrm{b}}$. The dashed line is derived from L3 data [13]; the three dotted lines are derived from DELPHI data at various center of mass energies (from top to bottom, $161 \mathrm{GeV}, 172 \mathrm{GeV}$, and $183 \mathrm{GeV}$ ). Fluctuations in the curves arise from fluctuations in the data.

constraining. This limit is almost an order of magnitude stronger than either the L3 or DELPHI limits on the same process.

The most stringent limits come from the OPAL data. Using Equations 4.1 and 4.2 , we translate these data into upper bounds on $N_{\mathrm{TC}} \mathcal{A}_{\gamma \gamma}$ and $N_{\mathrm{TC}} \mathcal{A}_{\mathrm{Z}^{0} \gamma}$. Assuming that $\mathrm{P}^{\mathrm{a}}$ decay to photons dominates, $\mathrm{BR}\left(\mathrm{P}^{\mathrm{a}} \rightarrow \gamma \gamma\right) \approx 1$ and $f_{\mathrm{Pa}}=123 \mathrm{GeV}$, we find $N_{\mathrm{TC}} \mathcal{A}_{\gamma \gamma}<15$ for $M_{\mathrm{Pa}}<M_{\mathrm{Z}^{0}}$; for $M_{\mathrm{Pa}}<140 \mathrm{GeV}$, we find that $N_{\mathrm{TC}} \mathcal{A}_{\gamma \gamma}<40$. We find $N_{\mathrm{TC}} \mathcal{A}_{\mathrm{Z}^{0} \gamma}<9$ for $M_{\mathrm{P}^{\mathrm{a}}}<M_{\mathrm{Z}^{0}}$; for $M_{\mathrm{P}^{\mathrm{a}}}<140 \mathrm{GeV}$, we find that $N_{\mathrm{TC}} \mathcal{A}_{\mathrm{Z}^{0} \gamma}<17$. For larger masses, both limits become rapidly less constraining. We plot our results based on the data from all three collaborations in Figure 8.

\subsubsection{Limits from $\mathrm{e}^{+} \mathrm{e}^{-} \rightarrow \gamma \mathrm{P}^{\mathrm{a}} \rightarrow \gamma \mathrm{b} \overline{\mathrm{b}}$}

If the PNGB decays predominantly to $b \bar{b}$ pairs, the final state can contain a hard photon, and two $b$ jets (Figure $2 \mathrm{a}$ with $\mathrm{P}^{\mathrm{a}} \rightarrow \mathrm{b} \overline{\mathrm{b}}$ ). The DELPHI and L3 collaborations have both published limits from their LEP II data samples.

The DELPHI collaboration has published limits on the production of a scalar resonance, $\mathrm{H}$, decaying to $b \bar{b}$ pairs. They have performed this analysis at each of three center of mass energies, based on $9.7 \mathrm{pb}^{-1}$ of data collected at $\sqrt{s}=161 \mathrm{GeV}, 10.1 \mathrm{pb}^{-1}$ of data collected at $\sqrt{s}=172 \mathrm{GeV}$, and $47.7 \mathrm{pb}^{-1}$ of data collected at $\sqrt{s}=183 \mathrm{GeV}$ [12]. They find no evidence for a new resonance, and place $95 \%$ c.l. upper limits on the cross section $\sigma\left(\mathrm{e}^{+} \mathrm{e}^{-} \rightarrow \mathrm{H} \gamma\right) \mathrm{BR}(\mathrm{H} \rightarrow \mathrm{b} \overline{\mathrm{b}})$, as a function of $M_{\mathrm{H}}$. Their highest-energy data is the most constraining; for $60 \mathrm{GeV}<M_{\mathrm{H}}<184 \mathrm{GeV}$, they find $\sigma\left(\mathrm{e}^{+} \mathrm{e}^{-} \rightarrow \mathrm{H} \gamma\right) \mathrm{BR}(\mathrm{H} \rightarrow \mathrm{b} \overline{\mathrm{b}})<0.50 \mathrm{pb}$, almost independent of $M_{\mathrm{H}}$.

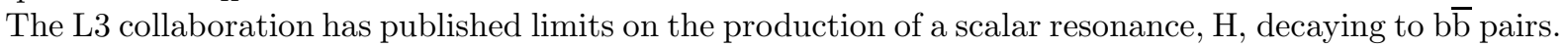
They have performed this analysis on $176 \mathrm{pb}^{-1}$ of data collected at $\sqrt{s}=189 \mathrm{GeV}$ [13]. They find no evidence for a new resonance, and place $95 \%$ c.l. upper limits on the cross section $\sigma\left(\mathrm{e}^{+} \mathrm{e}^{-} \rightarrow \mathrm{H} \gamma\right) \mathrm{BR}(\mathrm{H} \rightarrow \mathrm{b} \overline{\mathrm{b}})$, as a function of $M_{\mathrm{H}}$. For $70 \mathrm{GeV}<M_{\mathrm{H}}<170 \mathrm{GeV}$, they find $\sigma\left(\mathrm{e}^{+} \mathrm{e}^{-} \rightarrow \mathrm{H} \gamma\right) \mathrm{BR}(\mathrm{H} \rightarrow \mathrm{b} \overline{\mathrm{b}})<0.30 \mathrm{pb}$.

Using Equations 4.1 and 4.2 , we can translate this data into upper bounds on both $N_{\mathrm{TC}} \mathcal{A}_{\gamma \gamma}$ and $N_{\mathrm{TC}} \mathcal{A}_{\mathrm{Z}^{0} \gamma}$. Assuming the $\mathrm{P}^{\mathrm{a}}$ decays predominantly to $\mathrm{b} \overline{\mathrm{b}}$ jets, $\mathrm{BR}\left(\mathrm{P}^{\mathrm{a}} \rightarrow \mathrm{b} \overline{\mathrm{b}}\right) \approx 1$ and $f_{\mathrm{Pa}}=123 \mathrm{GeV}$, we find that $N_{\mathrm{TC}} \mathcal{A}_{\gamma \gamma}<62$ for $M_{\mathrm{P}^{\mathrm{a}}}<M_{\mathrm{Z}^{0}}$; for $M_{\mathrm{Pa}}<140 \mathrm{GeV}$, we find that $N_{\mathrm{TC}} \mathcal{A}_{\gamma \gamma}<140$. We find $N_{\mathrm{TC}} \mathcal{A}_{\mathrm{Z}^{0} \gamma}<30$ for $M_{\mathrm{P}^{\mathrm{a}}}<M_{\mathrm{Z}^{0}}$; for $M_{\mathrm{P}^{\mathrm{a}}}<140 \mathrm{GeV}$, we find that $N_{\mathrm{TC}} \mathcal{A}_{\mathrm{Z}^{0} \gamma}<60$. For larger masses, both limits become rapidly less constraining. We plot our results based on the data from both collaborations in Figure 9. 

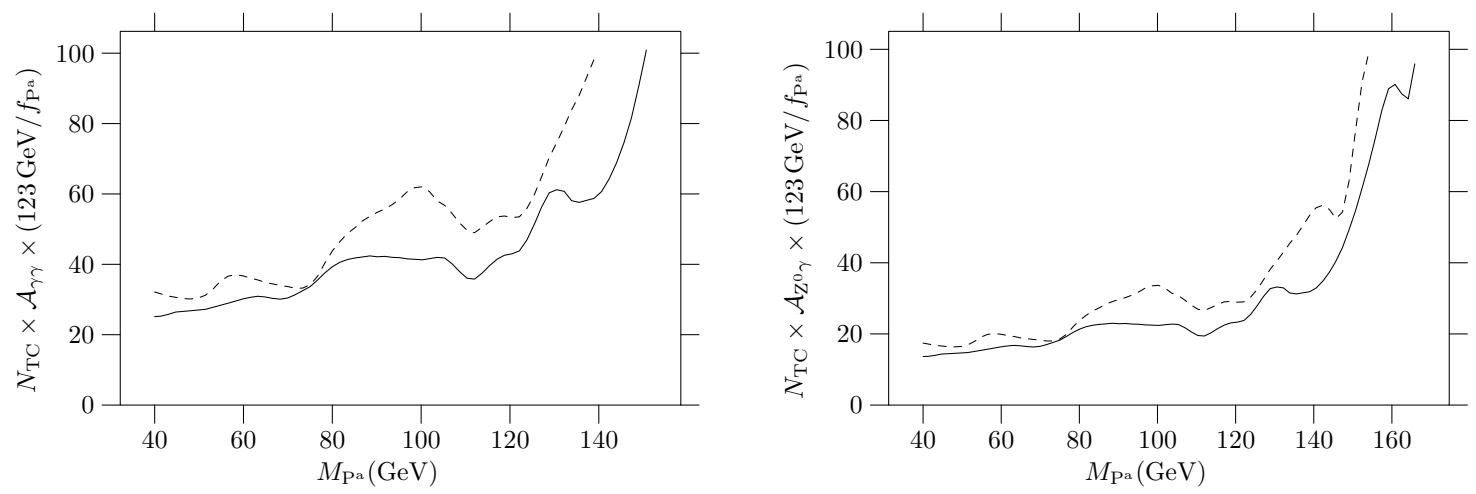

Figure 10: Upper limits at $95 \%$ c.l. on $N_{\mathrm{TC}} \mathcal{A}_{\gamma \gamma}\left(123 \mathrm{GeV} / f_{\mathrm{P}^{\mathrm{a}}}\right)$ (at left) and $N_{\mathrm{TC}} \mathcal{A}_{\mathrm{Z}^{0} \gamma}\left(123 \mathrm{GeV} / f_{\mathrm{P}^{a}}\right.$ ) (at right) from $\mathrm{e}^{+} \mathrm{e}^{-} \rightarrow \gamma \mathrm{P}^{\mathrm{a}} \rightarrow \gamma$ E. The results were derived from DELPHI data [14]; the solid lines come from the stronger limit derived by DELPHI, while the dashed lines correspond to the weaker limit. Fluctuations in the curves arise from fluctuations in the data.

\subsubsection{Limits from $\mathrm{e}^{+} \mathrm{e}^{-} \rightarrow \gamma \mathrm{P}^{\mathrm{a}} \rightarrow \gamma \mathrm{E}$}

If the predominant decays of the PNGB are invisible, we can find at LEP II a final state with a single hard photon and missing energy (Figure $2 \mathrm{a}$ with $\mathrm{P}^{\mathrm{a}} \rightarrow$ E). The DELPHI collaboration has searched for anomalous single photon events produced by a new scalar particle, $\mathrm{X}$, in $51 \mathrm{pb}^{-1}$ of data collected at $183 \mathrm{GeV}$ and in $158 \mathrm{pb}^{-1}$ collected at $189 \mathrm{GeV}$ [14]. They find no evidence for a new resonance, and place $95 \%$ c.l. upper limits on the production cross section $\sigma_{\mathrm{X}}$ as a function of $M_{\mathrm{X}}$. They provide two limits, based on their inclusion of data from different calorimeters: for $40 \mathrm{GeV}<M_{\mathrm{X}}<160 \mathrm{GeV}$, the stronger (weaker) limit is $\sigma_{\mathrm{X}}<0.2 \mathrm{pb}(0.3 \mathrm{pb})$.

Using Equations 4.1 and 4.2, we translate these data into upper bounds on $N_{\mathrm{TC}} \mathcal{A}_{\gamma \gamma}$ and $N_{\mathrm{TC}} \mathcal{A}_{\mathrm{Z}^{0} \gamma}$. Assuming that invisible decays of the $\mathrm{P}^{\mathrm{a}}$ dominate, $\mathrm{BR}\left(\mathrm{P}^{\mathrm{a}} \rightarrow \mathbb{E}\right) \approx 1$ and $f_{\mathrm{Pa}}=123 \mathrm{GeV}$, we find $N_{\mathrm{TC}} \mathcal{A}_{\gamma \gamma}<$ 40 for $M_{\mathrm{Pa}}<M_{\mathrm{Z}^{0}}$; for $M_{\mathrm{Pa}}<140 \mathrm{GeV}$, we find $N_{\mathrm{TC}} \mathcal{A}_{\gamma \gamma}<60$. We find $N_{\mathrm{TC}} \mathcal{A}_{\mathrm{Z}^{0} \gamma}<23$ for $M_{\mathrm{P}^{a}}<M_{\mathrm{Z}^{0}}$; for $M_{\mathrm{Pa}}<140 \mathrm{GeV}$, we find $N_{\mathrm{TC}} \mathcal{A}_{\mathrm{Z}^{0} \gamma}<33$. We plot our results in Figure 10 .

\subsection{Processes constraining both $N_{\mathrm{TC}} \mathcal{A}_{\mathrm{Z}^{0} \gamma}$ and $N_{\mathrm{TC}} \mathcal{A}_{\mathrm{Z}^{0} \mathrm{Z}^{0}}$}

In order to place limits on $\mathcal{A}_{\mathrm{Z}^{0} \mathrm{Z}^{0}}$ from LEP II data, we need to find states which include both intermediate and final $\mathrm{Z}^{0}$ bosons coupled to the $\mathrm{P}^{\mathrm{a}}$. Unfortunately, the most general processes that include these states also include three other diagrams, which receive contributions not only from $\mathcal{A}_{\mathrm{Z}^{0} \mathrm{Z}^{0}}$, but also from $\mathcal{A}_{\gamma \gamma}$ and $\mathcal{A}_{\mathrm{Z}^{0} \gamma}\left(\right.$ Figure 2c). ${ }^{4}$

In this section, we explore a restricted set of processes, those which include a real $\mathrm{Z}^{0}$ in the final state (Figure 2b). In the context of experiments, this involves requiring that the final state visible energy which is assumed not to come from the $\mathrm{P}^{\mathrm{a}}$ satisfies an invariant mass constraint, $M_{\mathrm{visible}} \approx M_{\mathrm{Z}^{0}}$. While this simplifies the analysis significantly, it reduces both the number of available published analyses, and the range of PNGB masses that are accessible, such that $M_{\mathrm{Pa}^{\mathrm{a}}}<\sqrt{s}-M_{\mathrm{Z}^{0}}$. The LEP II data collected at $\sqrt{s}=189 \mathrm{GeV}$ for example, can only probe PNGB masses lighter than about $95 \mathrm{GeV}$. 


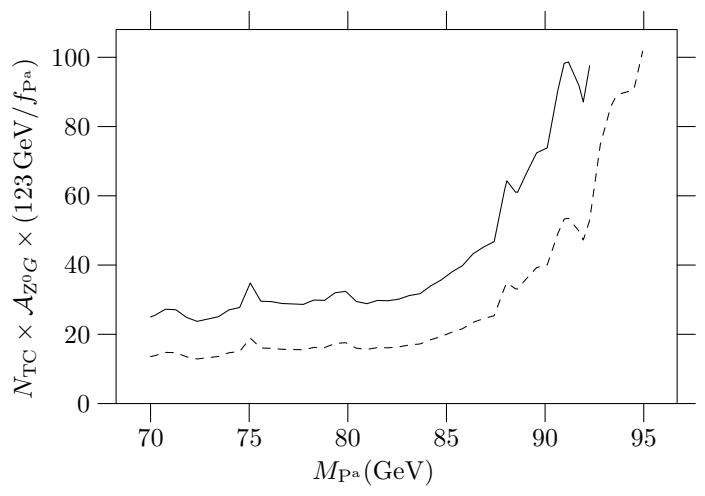

Figure 11: Upper limits at $95 \%$ c.l. on $N_{\mathrm{TC}} \mathcal{A}_{\mathrm{Z}^{0} \gamma}\left(123 \mathrm{GeV} / f_{\mathrm{P}^{\mathrm{a}}}\right)$ (solid line) and $N_{\mathrm{TC}} \mathcal{A}_{\mathrm{Z}^{0} \mathrm{Z}^{0}}\left(123 \mathrm{GeV} / f_{\mathrm{P}^{\mathrm{a}}}\right)$ (dashed line) from $\mathrm{e}^{+} \mathrm{e}^{-} \rightarrow \mathrm{Z}^{0} \mathrm{P}^{\mathrm{a}} \rightarrow \mathrm{f} \overline{\mathrm{f}} \mathrm{E}$, where the PNGB decays invisibly. The results were derived from ALEPH data [15]. Fluctuations in the curves arise from fluctuations in the data.

\subsubsection{Limits from $\mathrm{e}^{+} \mathrm{e}^{-} \rightarrow \mathrm{P}^{\mathrm{a}}\left(\gamma^{*} / \mathrm{Z}^{*}\right) \rightarrow \mathrm{f} \overline{\mathrm{f}} \mathrm{E}$}

If the PNGB is produced in association with a real $\mathrm{Z}^{0}$, the final state can contain missing energy from the PNGB decay, and two fermions from the $\mathrm{Z}^{0}$ decay (Figure $2 \mathrm{~b}$ ). The ALEPH collaboration has searched in this mode for the production of a scalar boson, $\mathrm{h}$, in $172 \mathrm{pb}^{-1}$ of data collected at $189 \mathrm{GeV}$ [15]. To insure that the visible energy comes from a $Z^{0}$, the collaboration requires that the invariant mass of the visible decay products approximately equal the invariant mass of the $\mathrm{Z}^{0}, M_{\mathrm{f} \overline{\mathrm{f}}} \approx M_{\mathrm{Z}^{0}}$. They find no evidence for a new resonance, and place $95 \%$ c.l. upper limits on the cross section for $\mathrm{hZ}^{0}$ production, scaled to the SM cross section, ${ }^{5}$ via $\mathrm{BR}(\mathrm{h} \rightarrow \mathrm{E}) \sigma\left(\mathrm{e}^{+} \mathrm{e}^{-} \rightarrow \mathrm{hZ} Z^{0}\right) / \sigma\left(\mathrm{e}^{+} \mathrm{e}^{-} \rightarrow \mathrm{hZ}\right)_{\mathrm{SM}}$, which we label $R$. For $M_{\mathrm{Pa}}<85 \mathrm{GeV}$, the upper limit is approximately $R<0.1$; for larger $\mathrm{P}^{\mathrm{a}}$ masses, the limit rises rapidly to $R<1$ at $M_{\mathrm{Pa}}=95 \mathrm{GeV}$.

Using Equations 4.3 and 4.4, we translate these data into upper bounds on $N_{\mathrm{TC}} \mathcal{A}_{\mathrm{Z}^{0} \gamma}$ and $N_{\mathrm{TC}} \mathcal{A}_{\mathrm{Z}^{0} \mathrm{Z}^{0}}$. Assuming the $\mathrm{P}^{\mathrm{a}}$ predominantly decays into invisible states and that $f_{\mathrm{Pa}^{\mathrm{a}}}=123 \mathrm{GeV}$, we find that $N_{\mathrm{TC}} \mathcal{A}_{\mathrm{Z}^{0} \gamma}<$ 20 for $M_{\mathrm{Pa}}<85 \mathrm{GeV}$, with the limit rapidly weakening for larger masses. For $M_{\mathrm{Pa}}<85 \mathrm{GeV}$, we find that $N_{\mathrm{TC}} \mathcal{A}_{\mathrm{Z}^{0} \mathrm{Z}^{0}}<30$. We plot our results in Figure 11

\subsubsection{Limits from $\mathrm{e}^{+} \mathrm{e}^{-} \rightarrow \mathrm{P}^{\mathrm{a}}\left(\gamma^{*} / \mathrm{Z}^{*}\right) \rightarrow \mathrm{f} \overline{\mathrm{f}} \gamma \gamma$}

If the PNGB is produced in association with a real $\mathrm{Z}^{0}$, the final state can contain two photons from the PNGB decay, and two fermions from the $\mathrm{Z}^{0}$ decay (Figure $2 \mathrm{~b}$ ). The L3 collaboration has searched in this mode for the production of a scalar boson, $\mathrm{h}$, in $176 \mathrm{pb}^{-1}$ of data collected at $189 \mathrm{GeV}$ [16]. The collaboration requires that the fermions come from a real $\mathrm{Z}^{0}$ by applying an invariant mass cut. They find no evidence for a new resonance, and place $95 \%$ c.l. upper limits on the cross section for $\mathrm{hZ}^{0}$ production, scaled to the SM cross section (given in the previous section), via $R=\mathrm{BR}(\mathrm{h} \rightarrow \gamma \gamma) \sigma\left(\mathrm{e}^{+} \mathrm{e}^{-} \rightarrow \mathrm{hZ}{ }^{0}\right) / \sigma\left(\mathrm{e}^{+} \mathrm{e}^{-} \rightarrow \mathrm{hZ}^{0}\right)_{\mathrm{SM}}$. For $M_{\mathrm{Pa}}<85 \mathrm{GeV}$, the upper limit is approximately $R<0.1$; for larger masses, the limit rises rapidly to $R<1$ at $M_{\mathrm{Pa}}=98 \mathrm{GeV}$.

\footnotetext{
${ }^{4}$ This is not an issue for SM Higgs searches, since there are no tree level couplings of the Higgs to photons.

${ }^{5}$ The $\mathrm{SM} \mathrm{e} \mathrm{e}^{+} \rightarrow \mathrm{HZ}^{0}$ cross section can be found in the literature [25, 26, 27]

$$
\sigma\left(\mathrm{e}^{+} \mathrm{e}^{-} \rightarrow \mathrm{HZ}^{0}\right)=\frac{\pi \alpha_{\mathrm{em}}^{2}\left(1+\left(1-4 s_{W}^{2}\right)^{2}\right)}{192 s_{W}^{4} c_{W}^{4} s^{2}\left(s-M_{\mathrm{Z}^{0}}^{2}\right)^{2}} \Lambda^{1 / 2}\left(\Lambda+12 s M_{\mathrm{Z}^{0}}^{2}\right),
$$
}

where $\Lambda=\lambda\left(s, M_{\mathrm{Z}^{0}}^{2}, M_{\mathrm{H}}^{2}\right)$, as defined in Section 2 . 


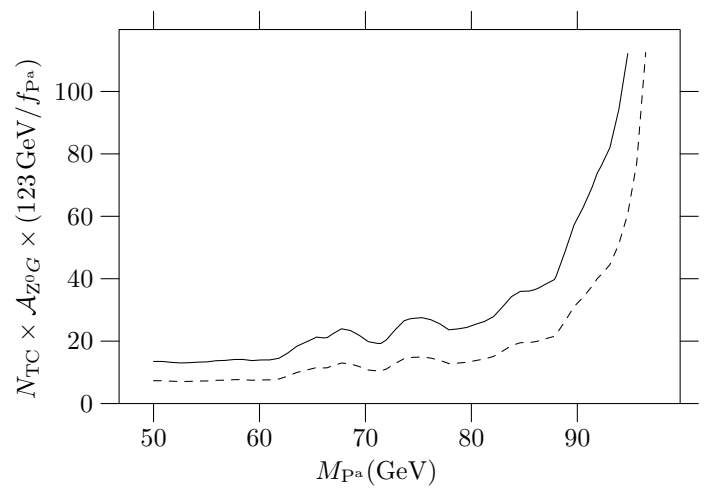

Figure 12: Upper limits at $95 \%$ c.l. on $N_{\mathrm{TC}} \mathcal{A}_{\mathrm{Z}^{0} \gamma}\left(123 \mathrm{GeV} / f_{\mathrm{P}^{\mathrm{a}}}\right)$ (solid line) and $N_{\mathrm{TC}} \mathcal{A}_{\mathrm{Z}^{0} \mathrm{Z}^{0}}\left(123 \mathrm{GeV} / f_{\mathrm{Pa}^{\mathrm{a}}}\right)$ (dashed line) from $\mathrm{e}^{+} \mathrm{e}^{-} \rightarrow \mathrm{Z}^{0} \mathrm{P}^{\mathrm{a}} \rightarrow \mathrm{f} \overline{\mathrm{f}} \gamma \gamma$. The results were derived from L3 data [16]. Fluctuations in the curves arise from fluctuations in the data.

Using Equation 4.3 and 4.4, we translate these data into upper bounds on $N_{\mathrm{TC}} \mathcal{A}_{\mathrm{Z}^{0} \gamma}$ and $N_{\mathrm{TC}} \mathcal{A}_{\mathrm{Z}^{0} \mathrm{Z}^{0}}$. Assuming the $\mathrm{P}^{\mathrm{a}}$ predominantly decays into photon pairs and $f_{\mathrm{P}^{\mathrm{a}}}=123 \mathrm{GeV}$, we find that $N_{\mathrm{TC}} \mathcal{A}_{\mathrm{Z}^{0} \gamma}<15$ for $M_{\mathrm{Pa}}<85 \mathrm{GeV}$, with the limit rapidly weakening for larger masses. For $M_{\mathrm{Pa}}<85 \mathrm{GeV}$, we find that $N_{\mathrm{TC}} \mathcal{A}_{\mathrm{Z}^{0} \mathrm{Z}^{0}}<25$. We plot our results in Figure 12 .

\subsection{Process $\mathrm{e}^{+} \mathrm{e}^{-} \rightarrow \mathrm{P}^{\mathrm{a}} \mathrm{e}^{+} \mathrm{e}^{-}$constraining $N_{\mathrm{TC}} \mathcal{A}_{\gamma \gamma}$}

Since the $\mathrm{P}^{\mathrm{a}}$ couples to the electroweak gauge bosons, it is possible to produce them in the $2 \rightarrow 3$ interaction, $\mathrm{e}^{+} \mathrm{e}^{-} \rightarrow \mathrm{P}^{\mathrm{a}} \mathrm{e}^{+} \mathrm{e}^{-}$(Figure 2d). The L3 collaboration has performed a search for anomalous couplings of a SM Higgs boson, $\mathrm{H}$, to electroweak gauge bosons in $176 \mathrm{pb}^{-1}$ of data collected at $189 \mathrm{GeV}$ [13]. They find no evidence for such anomalous couplings, and place $95 \%$ c.l. upper limits on the decay widths $\Gamma_{\mathrm{b}}=\Gamma(\mathrm{H} \rightarrow \gamma \gamma) \mathrm{BR}(\mathrm{H} \rightarrow \mathrm{b} \overline{\mathrm{b}})$ and $\Gamma_{\gamma \gamma}=\Gamma(\mathrm{H} \rightarrow \gamma \gamma) \mathrm{BR}(\mathrm{H} \rightarrow \gamma \gamma)$, as a function of $M_{\mathrm{H}}$. They find $\Gamma_{\gamma \gamma}<10^{-1} \mathrm{MeV}$ for $M_{\mathrm{H}}<70 \mathrm{GeV}$, rising to $\Gamma_{\gamma \gamma}<10^{2} \mathrm{GeV}$ at $M_{\mathrm{H}}<170 \mathrm{GeV}$; the limits on $\Gamma_{\mathrm{bb}}$ are approximately an order of magnitude larger at all $M_{\mathrm{H}}$.

Using Equation 2.15, we translate these data into upper bounds on ${ }^{6} N_{\mathrm{TC}} \mathcal{A}_{\gamma \gamma}$. Assuming the photon decay mode of the $\mathrm{P}^{\mathrm{a}}$ dominates and $f_{\mathrm{P}^{\mathrm{a}}}=123 \mathrm{GeV}$, we find $N_{\mathrm{TC}} \mathcal{A}_{\gamma \gamma}<5$ for $M_{\mathrm{P}^{\mathrm{a}}}<M_{\mathrm{Z}^{0}}$; for $M_{\mathrm{P}^{\mathrm{a}}}<140 \mathrm{GeV}$, we find $N_{\mathrm{TC}} \mathcal{A}_{\gamma \gamma}<10$. If instead the $\mathrm{P}^{\mathrm{a}}$ decays predominantly to $\mathrm{b} \overline{\mathrm{b}}$, we find $N_{\mathrm{TC}} \mathcal{A}_{\gamma \gamma}<12$ for $M_{\mathrm{P}^{\mathrm{a}}}<M_{\mathrm{Z}^{0}}$, and $N_{\mathrm{TC}} \mathcal{A}_{\gamma \gamma}<20$ for $M_{\mathrm{Pa}}<140 \mathrm{GeV}$. We plot our results in Figure 13 .

\section{Summarizing the LEP I and LEP II Limits}

In this section, we summarize and compare the limits derived from the LEP I and LEP II data sets. First, we graphically examine the region of overlap between the LEP I and LEP II data sets. Then, we tabulate our derived limits on the various anomaly factors.

Both LEP I and LEP II provide access to $\mathcal{A}_{\mathrm{Z}^{0} \gamma}$ and $\mathcal{A}_{\mathrm{Z}^{0} \mathrm{Z}^{0}}$. In Figure 14, we display the region of overlap between the $\mathcal{A}_{\mathrm{Z}^{0} \gamma}$ results from LEP I and the $\mathcal{A}_{\mathrm{Z}^{0} \gamma}$-dominated limits from LEP II. We find that for all decay modes (except $\mathrm{P}^{\mathrm{a}} \rightarrow \mathrm{jj}$, which is not probed at LEP II), the LEP I data provide a much stronger limit than the LEP II data for $M_{\mathrm{Pa}}<80 \mathrm{GeV}$, while for $M_{\mathrm{Pa}}>80 \mathrm{GeV}$, the LEP II data take over. Figure 15

\footnotetext{
${ }^{6}$ The upper bound is only on $\mathcal{A}_{\gamma \gamma}$, rather than some combination of $\mathcal{A}_{\gamma \gamma}, \mathcal{A}_{\mathrm{Z}^{0} \gamma}$ and $\mathcal{A}_{\mathrm{Z}^{0} \mathrm{Z}^{0}}$, because kinematic factors ensure that the gauge bosons internal to the $2 \rightarrow 3$ process are predominantly photons [4, 13].
} 


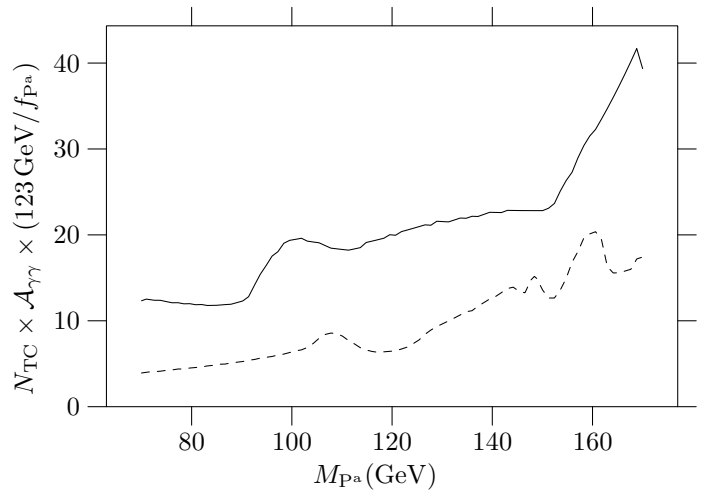

Figure 13: Upper limits at $95 \%$ c.l. on $N_{\mathrm{TC}} \mathcal{A}_{\gamma \gamma}\left(123 \mathrm{GeV} / f_{\mathrm{Pa}}\right)$ from $\mathrm{e}^{+} \mathrm{e}^{-} \rightarrow \mathrm{P}^{\mathrm{a}} \mathrm{e}^{+} \mathrm{e}^{-}$, derived from L3 data. The solid curve holds if $\mathrm{BR}\left(\mathrm{P}^{\mathrm{a}} \rightarrow \mathrm{b} \overline{\mathrm{b}}\right) \approx 1$, while the dashed curve holds if $\mathrm{BR}\left(\mathrm{P}^{\mathrm{a}} \rightarrow \gamma \gamma\right) \approx 1$. Fluctuations in the curves arise from fluctuations in the data.

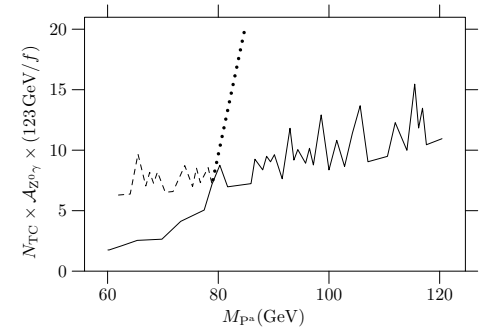

(a) $\mathrm{P}^{\mathrm{a}} \rightarrow \gamma \gamma$

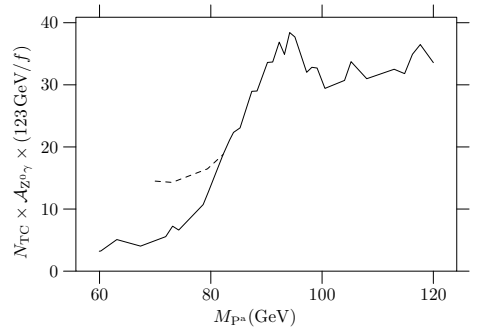

(b) $\mathrm{P}^{\mathrm{a}} \rightarrow \mathrm{b} \overline{\mathrm{b}}$

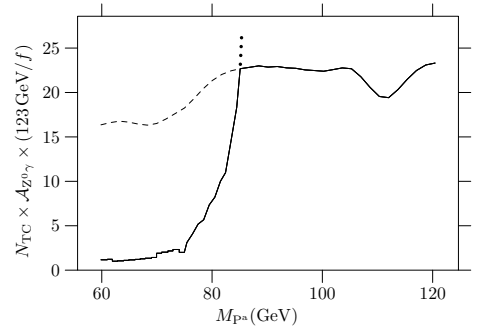

(c) $\mathrm{P}^{\mathrm{a}} \rightarrow \mathrm{E}$

Figure 14: Combined LEP I and LEP II upper limits at $95 \%$ c.l. on $N_{\mathrm{TC}} \mathcal{A}_{\mathrm{Z}^{0} \gamma}\left(123 \mathrm{GeV} / f_{\mathrm{Pa}}\right)$ from $\gamma \gamma \gamma$ (left), $\gamma \mathrm{b} \overline{\mathrm{b}}$ (center), and $\gamma \mathrm{E}$ (right) final states, for $M_{\mathrm{P}^{\mathrm{a}}}$ within $30 \mathrm{GeV}$ of $M_{\mathrm{Z}^{0}}$. In each case, the solid line indicates the combined limit, the dotted line indicates the LEP I limit, and the dashed line indicates the LEP II limit. 


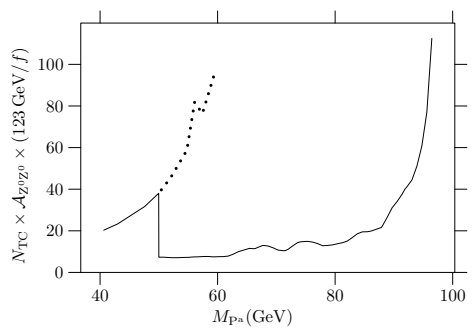

(a) $\mathrm{Pa}^{\mathrm{a}} \rightarrow \gamma \gamma$

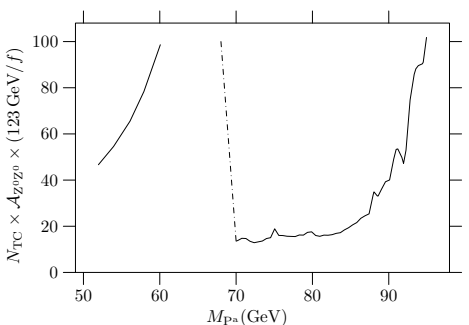

(b) $\mathrm{Pa}^{\mathrm{a}} \rightarrow \mathrm{E}$

Figure 15: Combined LEP I and LEP II upper limits at $95 \%$ c.l. on $N_{\mathrm{TC}} \mathcal{A}_{\mathrm{Z}^{0} \mathrm{Z}^{0}}\left(123 \mathrm{GeV} / f_{\mathrm{P}^{\mathrm{a}}}\right)$ from $\mathrm{P}^{\mathrm{a}} \rightarrow \gamma \gamma$ (at left) and $\mathrm{P}^{\mathrm{a}} \rightarrow \mathbb{E}$ (at right). In each case, the solid limit indicates the combined limit and the dotted line indicates the LEP I limits. The dotted-dashed line in the right hand plot is only to guide the eye since the data sets sensitive to low-mass (LEP I) and high-mass (LEP II) PNGBs do not overlap).

Table 1: Upper limits on $N_{\mathrm{TC}} \mathcal{A}_{G_{1} G_{2}}\left(123 \mathrm{GeV} / f_{\mathrm{P}^{a}}\right)$ from the LEP I and LEP II data samples. The limits in this table are independent of each other, and can be applied directly to any model.

\begin{tabular}{|c|c|c|c|c|c|c|c|c|c|c|c|c|}
\hline PNGB & \multicolumn{4}{|c|}{ produced via $\mathcal{A}_{\gamma \gamma}$} & \multicolumn{4}{|c|}{ produced via $\mathcal{A}_{\mathrm{Z}^{0} \gamma}$} & \multicolumn{4}{|c|}{ produced via $\mathcal{A}_{\mathrm{Z}^{0} \mathrm{Z}^{0}}$} \\
\hline mass & \multicolumn{4}{|c|}{ decay mode } & \multicolumn{4}{|c|}{ decay mode } & \multicolumn{4}{|c|}{ decay mode } \\
\hline$M_{\mathrm{Pa}} \leq$ & $\mathrm{E}$ & $\gamma \gamma$ & $\mathrm{bb}$ & $\mathrm{jj}$ & $\mathrm{E}$ & $\overline{\gamma \gamma}$ & $\mathrm{bb}$ & $\mathrm{jj}$ & $\mathrm{E}$ & $\gamma \gamma$ & $\mathrm{bb}$ & $\mathrm{jj}$ \\
\hline $30 \mathrm{GeV}$ & & & & & 0.63 & 0.75 & 1.1 & 1.3 & 13 & 12 & & 50 \\
\hline $60 \mathrm{GeV}$ & & & & & 1.2 & 1.8 & 3.2 & 3.1 & & & & \\
\hline $80 \mathrm{GeV}$ & & 5 & 12 & & 7.8 & 10 & 14 & 13 & & & & \\
\hline $100 \mathrm{GeV}$ & & 6 & 19 & & & & & & & & & \\
\hline $120 \mathrm{GeV}$ & & 7 & 20 & & & & & & & & & \\
\hline $140 \mathrm{GeV}$ & & 13 & 23 & & & & & & & & & \\
\hline $160 \mathrm{GeV}$ & & 19 & 32 & & & & & & & & & \\
\hline
\end{tabular}


Table 2: Upper limits on $N_{\mathrm{TC}} \mathcal{A}_{G_{1} G_{2}}\left(123 \mathrm{GeV} / f_{\mathrm{Pa}}\right)$ from the LEP I and LEP II data samples. The limits in this table are not independent: a given final state simultaneously provides two limits: for either $\mathcal{A}_{\gamma \gamma}$ and $\mathcal{A}_{Z^{0} \gamma}$ or $\mathcal{A}_{Z^{0} \gamma}$ and $\mathcal{A}_{Z^{0} Z^{0}}$. For a given mass and $\mathrm{P}^{a}$ decay mode, the related limits on $\mathcal{A}_{\gamma \gamma}$ and $\mathcal{A}_{Z^{0} \gamma}\left[\mathcal{A}_{Z^{0} \gamma}\right.$ and $\mathcal{A}_{\mathrm{Z}^{0} \mathrm{Z}^{0}}$ ] are surrounded by parentheses [brackets]; e.g. for an $80 \mathrm{GeV}$ PNGB decaying to two photons, the limits (14) and (8) are related, as are the limits [25] and [14]. Each limit in this table is derived under the assumption that its production process (anomaly factor) dominates, as discussed in 4 . For models in which the various anomaly factors are of quite different sizes (as in all models studied in Section 6), the strongest limit from the table applies directly. For a model in which the two related production modes are comparable, the limits can be combined as discussed in Section 4 to obtain a stronger bound on $N_{\mathrm{TC}}$.

\begin{tabular}{|c|c|c|c|c|c|c|c|c|c|c|c|c|}
\hline PNGB & \multicolumn{4}{|c|}{ produced via $\mathcal{A}_{\gamma \gamma}$} & \multicolumn{4}{|c|}{ produced via $\mathcal{A}_{\mathrm{Z}^{0} \gamma}$} & \multicolumn{4}{|c|}{ produced via $\mathcal{A}_{\mathrm{Z}^{0} \mathrm{Z}^{0}}$} \\
\hline mass & \multicolumn{4}{|c|}{ decay mode } & \multicolumn{4}{|c|}{ decay mode } & \multicolumn{4}{|c|}{ decay mode } \\
\hline$M_{\mathrm{Pa}} \leq$ & E & $\gamma \gamma$ & $\overline{b b}$ & $\mathrm{jj}$ & E & $\gamma \gamma$ & $\mathrm{b} \bar{b}$ & $\mathrm{jj}$ & $\mathrm{E}$ & $\overline{\gamma \gamma}$ & $\mathrm{bb}$ & $\overline{\mathrm{jj}}$ \\
\hline $30 \mathrm{GeV}$ & & (13) & & & & $(8)$ & & & & & & \\
\hline $60 \mathrm{GeV}$ & $(30)$ & (12) & & & (16) & $(7) /[14]$ & & & & {$[8]$} & & \\
\hline $80 \mathrm{GeV}$ & (39) & (14) & (30) & & $(21) /[30]$ & $(8) /[25]$ & (17) & & {$[16]$} & {$[14$} & & \\
\hline $100 \mathrm{GeV}$ & $(42)$ & (18) & $(58)$ & & $(23)$ & (10) & $(32)$ & & & & & \\
\hline $120 \mathrm{GeV}$ & $(44)$ & $(22)$ & $(66)$ & & (23) & (12) & (36) & & & & & \\
\hline $140 \mathrm{GeV}$ & $(60)$ & $(36)$ & (98) & & (33) & $(20)$ & (53) & & & & & \\
\hline $160 \mathrm{GeV}$ & (165) & $(82)$ & (172) & & (86) & $(45)$ & (100) & & & & & \\
\hline
\end{tabular}

similarly displays the limited region of overlap between the $\mathcal{A}_{\mathrm{Z}^{0} \mathrm{Z}^{0}}$ dominated results from LEP I and the $\mathcal{A}_{\mathrm{Z}^{0} \mathrm{Z}^{0}}$ dominated limits from LEP II. Here, the LEP I data exist only up to $M_{\mathrm{Pa}} \approx 60 \mathrm{GeV}$ and the LEP II data are stronger than LEP I data where they exist.

Tables 1 and 2 gather the best limits on $N_{\mathrm{TC}} \mathcal{A}_{G_{1} G_{2}}\left(123 \mathrm{GeV} / f_{\mathrm{Pa}}\right)$ from the experiments discussed in Sections 3 and 4 . In Table 1, we gather all limits that can be independently applied to TC models; that is, these limits are not directly linked with any other anomaly factor limits. In Table 2, we gather all limits that can not be independently applied; that is, the limits on $\mathcal{A}_{\gamma \gamma}$ and $\mathcal{A}_{\mathrm{Z}^{0} \mathrm{Z}^{0}}$ in this table are related to the corresponding limits on $\mathcal{A}_{\mathrm{Z}^{0} \gamma}$, as discussed in Section 4. In particular, it is permissible to apply these limits directly only if the appropriate anomaly factor dominates the $\mathrm{P}^{\mathrm{a}}$ production (as in the models we examine in Section 6).

\section{Implications for Technicolor Models}

In this section, we discuss how our limits on $\mathrm{P}^{\mathrm{a}}$ couplings constrain several classes of technicolor models. We begin with a quick look at the familiar one-family technicolor models in order to assess what properties a model must have in order that our limits constrain the masses of the PNGBs in that model. We then examine three other scenarios: near-critical Extended Technicolor models, models with weak isotriplet technifermions, and low-scale models. Because the data are sensitive to the ratio $N_{\mathrm{TC}} \mathcal{A}_{G_{1} G_{2}} / f_{\mathrm{Pa}}$ (per Equations 2.5 and 2.6), models with smaller technipion decay constants or larger anomaly factors will be more tightly constrained.

\subsection{One-family Technicolor Models}

The minimal one-family technicolor model of Farhi and Susskind [28] is a classic example of a technicolor model with PNGBs. The model contains one color singlet technilepton doublet, L, and one color triplet tech- 
Table 3: Upper limits on the number of technicolors, $N_{\mathrm{TC}}$, as a function of $\mathrm{P}^{\mathrm{a}}$ decay constant and PNGB mass in the Applequist-Terning one-family technicolor model of Section 6.1. The superscripted labels indicate the data used to calculate the limits: $\dagger: \mathcal{A}_{\gamma \gamma}$ from Table $1 ; \ddagger: \mathcal{A}_{Z^{0} \gamma}$ from Table $1 ; \$: \mathcal{A}_{Z^{0} Z^{0}}$ from Table 1; \#: $\mathcal{A}_{\gamma \gamma}$ from Table 2 .

\begin{tabular}{|c|c|c|}
\hline & \multicolumn{2}{|c|}{$N_{\mathrm{TC}} \leq$} \\
\hline$M_{\mathrm{Pa}} \leq$ & $\mathrm{P}_{\mathrm{E}} \rightarrow \gamma \gamma$ & $\mathrm{P}_{\mathrm{N}} \rightarrow \not \mathrm{E}$ \\
\hline $30 \mathrm{GeV}$ & $37 f_{\mathrm{PE}_{\mathrm{E}}} / v^{\#}$ & $295 f_{\mathrm{P}_{\mathrm{N}}} / v^{\$}$ \\
$60 \mathrm{GeV}$ & $34 f_{\mathrm{P}_{\mathrm{E}}} / v^{\#}$ & - \\
$80 \mathrm{GeV}$ & $14 f_{\mathrm{P}_{\mathrm{E}}} / v^{\dagger}$ & $364 f_{\mathrm{P}_{\mathrm{N}}} / v^{\$}$ \\
$100 \mathrm{GeV}$ & $17 f_{\mathrm{P}_{\mathrm{E}}} / v^{\dagger}$ & - \\
$120 \mathrm{GeV}$ & $20 f_{\mathrm{P}_{\mathrm{E}}} / v^{\dagger}$ & - \\
$140 \mathrm{GeV}$ & $37 f_{\mathrm{P}_{\mathrm{E}}} / v^{\dagger}$ & - \\
$160 \mathrm{GeV}$ & $54 f_{\mathrm{P}_{\mathrm{E}}} / v^{\dagger}$ & - \\
\hline
\end{tabular}

niquark doublet, Q, while the right-handed technifermions are all electroweak singlets. From Equation 2.3, we find $f=v / 2=123 \mathrm{GeV}$. The neutral PNGBs, described in terms of their technifermion quantum numbers and normalized as in Equation 2.11 are given by

$$
P^{1}=\frac{1}{4 \sqrt{3}}\left(3 \overline{\mathrm{L}} \gamma_{5} \mathrm{~L}-\overline{\mathrm{Q}} \gamma_{5} \mathrm{Q}\right) \quad P^{3}=\frac{1}{2 \sqrt{3}}\left(3 \overline{\mathrm{L}} \gamma_{5} \tau^{3} \mathrm{~L}-\overline{\mathrm{Q}} \gamma_{5} \tau^{3} \mathrm{Q}\right)
$$

These PNGBs decay dominantly in the two jets mode, either to q $\bar{q}$ via Extended Technicolor gauge bosons or QCD gluons, or in the case of the $\mathrm{P}^{1}$, by direct decays to gluon pairs [3, 28]. Therefore, the limits on $N_{\mathrm{TC}} \mathcal{A}_{\mathrm{Z}^{0} \gamma}$ and $N_{\mathrm{TC}} \mathcal{A}_{\mathrm{Z}^{0} \mathrm{Z}^{0}}$ from hadronic scalar decays (the jj modes from Tables 1 and 2) apply. Because the anomaly factors for these PNGBs (from Equations 2.13 and 2.14) are rather small,

$$
\begin{aligned}
& \mathcal{A}_{\gamma \gamma}^{1}=\frac{1}{3 \sqrt{3}} \approx 0.192 \quad \mathcal{A}_{\mathrm{Z}^{0} \gamma}^{1}=\frac{1}{3 \sqrt{3}} s_{W}^{2} \approx 0.044 \quad \mathcal{A}_{\mathrm{Z}^{0} \mathrm{Z}^{0}}^{1}=\frac{1}{3 \sqrt{3}} s_{W}^{4} \approx 0.010 \\
& \mathcal{A}_{\gamma \gamma}^{3}=\frac{1}{\sqrt{3}} \approx 0.577 \quad \mathcal{A}_{\mathrm{Z}^{0} \gamma}^{3}=\frac{1}{4 \sqrt{3}}\left(1-4 s_{W}^{2}\right) \approx 0.012 \quad \mathcal{A}_{\mathrm{Z}^{0} \mathrm{Z}^{0}}^{3}=\frac{1}{2 \sqrt{3}} s_{W}^{2}\left(1-2 s_{W}^{2}\right) \approx 0.036 .
\end{aligned}
$$

one obtains only weak limits on the size of the technicolor group; e.g. for $M_{\mathrm{P}^{1}} \leq 30 \mathrm{GeV}$, one has $N_{\mathrm{TC}} \leq 30$. The constraints derived from $\mathcal{A}_{\mathrm{Z}^{0} \mathrm{Z}^{0}}$ are even weaker. The results for the light PNGB $P^{0}=\left(3 \overline{\mathrm{E}} \gamma_{5} \mathrm{E}-\right.$ $\left.\overline{\mathrm{D}} \gamma_{5} \mathrm{D}\right) / \sqrt{24}$ in the model of Casalbuoni et. al. [5] are very similar, since the anomaly factors are equally small and $f_{\mathrm{Pa}}=123 \mathrm{GeV}$.

The one-family technicolor model of Applequist and Terning [23] includes PNGBs with $f_{\mathrm{Pa}}<v / 2$. This model was designed as an example of a realistic technicolor scenario that reduced the estimated technicolor contributions to the $S$ and $T$ parameters. QCD interactions and near-critical Extended Technicolor interactions combine to violate isospin symmetry strongly, and enhance quark and techniquark masses relative to lepton and technilepton masses. In the limit of extreme isospin breaking, the techniquarks dominate the Goldstone bosons eaten by the electroweak gauge bosons, leaving two light, non-degenerate neutral PNGBs composed mostly of technileptons,

$$
P_{\mathrm{N}}=\frac{1}{\sqrt{2}} \overline{\mathrm{N}} \gamma_{5} \mathrm{~N} \quad P_{\mathrm{E}}=\frac{1}{\sqrt{2}} \overline{\mathrm{E}} \gamma_{5} \mathrm{E},
$$


Table 4: Upper limits on the number of technicolors, $N_{\mathrm{TC}}$, as a function of the technifermion hypercharge $y$ and PNGB mass in the Manohar-Randall one-family weak-isotriplet TC model [1] of Section 6.1. Limits are shown for the cases where the dominant decays are invisible, two-photon, or $b \bar{b}$. The superscripted labels indicate the data used to calculate the limits: $\dagger: \mathcal{A}_{\gamma \gamma}$ from Table $1 ; \ddagger: \mathcal{A}_{Z^{0} \gamma}$ from Table $1 ; \#: \mathcal{A}_{\gamma \gamma}$ from Table 2 .

\begin{tabular}{|c|c|c|c|c|}
\hline \multicolumn{2}{|c|}{$\overline{\mathrm{PNGB}}$} & \multicolumn{3}{|c|}{$N_{\mathrm{TC}} \leq$} \\
\hline & $M_{\mathrm{Pa}} \leq$ & $\overline{\mathrm{P}^{\mathrm{a}} \rightarrow \mathrm{E}}$ & $\mathrm{P}^{\mathrm{a}} \rightarrow \gamma \gamma$ & $\mathrm{P}^{\mathrm{a}} \rightarrow \mathrm{b} \bar{b}$ \\
\hline \multirow{7}{*}{$\mathrm{P}^{1}$} & $30 \mathrm{GeV}$ & $0.16 / y^{2} \mp$ & $0.19 / y^{2} \ddagger$ & $0.28 / y^{2} \mp$ \\
\hline & $60 \mathrm{GeV}$ & $0.31 / y^{2} \ddagger$ & $0.44 / y^{2} \ddagger$ & $0.82 / y^{2} \ddagger$ \\
\hline & $80 \mathrm{GeV}$ & $2.00 / y^{2} \ddagger$ & $0.29 / y^{2 \dagger}$ & $0.71 / y^{2 \dagger}$ \\
\hline & $100 \mathrm{GeV}$ & $2.47 / y^{2} \#$ & $0.35 / y^{2 \dagger}$ & $1.12 / y^{2 \dagger}$ \\
\hline & $120 \mathrm{GeV}$ & $2.59 / y^{2} \#$ & $0.41 / y^{2 \dagger}$ & $1.18 / y^{2 \dagger}$ \\
\hline & $140 \mathrm{GeV}$ & $3.54 / y^{2} \#$ & $0.77 / y^{2 \dagger}$ & $1.36 / y^{2 \dagger}$ \\
\hline & $160 \mathrm{GeV}$ & $9.72 / y^{2} \#$ & $1.12 / y^{2 \dagger}$ & $1.89 / y^{2 \dagger}$ \\
\hline \multirow{7}{*}{$\mathrm{P}^{3}$} & $30 \mathrm{GeV}$ & 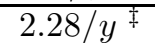 & $0.94 / y \#$ & $3.96 / y^{\ddagger}$ \\
\hline & $60 \mathrm{GeV}$ & $2.17 / y$ \# & $0.87 / y$ \# & $11.5 / y^{\ddagger}$ \\
\hline & $80 \mathrm{GeV}$ & $2.81 / y^{\#}$ & $0.36 / y^{\dagger}$ & $0.87 / y^{\dagger}$ \\
\hline & $100 \mathrm{GeV}$ & $3.03 / y$ \# & $0.43 / y^{\dagger}$ & $1.37 / y^{\dagger}$ \\
\hline & $120 \mathrm{GeV}$ & $3.18 / y$ \# & $0.51 / y^{\dagger}$ & $1.44 / y^{\dagger}$ \\
\hline & $140 \mathrm{GeV}$ & $4.33 / y^{\#}$ & $0.94 / y^{\dagger}$ & $1.66 / y^{\dagger}$ \\
\hline & $160 \mathrm{GeV}$ & $11.5 / y$ \# & $1.37 / y^{\dagger}$ & $2.31 / y^{\dagger}$ \\
\hline \multirow{7}{*}{$\mathrm{P}_{+}^{5}$} & $30 \mathrm{GeV}$ & $1.01^{\ddagger}$ & $1.20^{\ddagger}$ & - \\
\hline & $60 \mathrm{GeV}$ & $1.92 \ddagger$ & $2.72 \stackrel{\ddagger}{\ddagger}$ & - \\
\hline & $80 \mathrm{GeV}$ & $12.5^{\ddagger}$ & $2.16^{\dagger}$ & 一 \\
\hline & $100 \mathrm{GeV}$ & $18.2^{\#}$ & $2.60^{\dagger}$ & - \\
\hline & $120 \mathrm{GeV}$ & 19.0 \# & $3.03^{\dagger}$ & - \\
\hline & $140 \mathrm{GeV}$ & 26.0 \# & $5.63^{\dagger}$ & - \\
\hline & $160 \mathrm{GeV}$ & $71.4^{\#}$ & $8.23^{\dagger}$ & - \\
\hline
\end{tabular}


with separate decay constants, $f_{\mathrm{N}}<f_{\mathrm{E}}$. The anomaly factors for these PNGBs are not large

$$
\begin{gathered}
\mathcal{A}_{\gamma \gamma}^{\mathrm{N}}=\mathcal{A}_{\mathrm{Z}^{0} \gamma}^{\mathrm{N}}=0 \quad \mathcal{A}_{\mathrm{Z}^{0} \mathrm{Z}^{0}}^{\mathrm{N}}=\frac{1}{8 \sqrt{2}} \approx 0.088 \\
\mathcal{A}_{\gamma \gamma}^{\mathrm{E}}=\frac{1}{\sqrt{2}} \approx 0.707 \quad \mathcal{A}_{\mathrm{Z}^{0} \gamma}^{\mathrm{E}}=\frac{1}{4 \sqrt{2}}\left(1-4 s_{W}^{2}\right) \approx 0.014 \quad \mathcal{A}_{\mathrm{Z}^{0} \mathrm{Z}^{0}}^{\mathrm{E}}=\frac{1}{8 \sqrt{2}}\left(4 s_{W}^{4}+\left(1-2 s_{W}^{2}\right)^{2}\right) \approx 0.044 .
\end{gathered}
$$

In the most optimistic cases where $\mathrm{P}_{\mathrm{E}} \rightarrow \gamma \gamma$ and $\mathrm{P}_{\mathrm{N}} \rightarrow \notin$ are the dominant decay modes, the limits from Tables 1 and 2 yield the results on $N_{\mathrm{TC}}$ shown in Table 3 . Since the anomaly factor for $\mathrm{P}_{\mathrm{N}}$ is so small, the limits on $\mathrm{P}_{\mathrm{N}}$ would be phenomenologically relevant only if $f_{\mathrm{P}_{\mathrm{N}}} \lesssim v / 25$. The limits on $\mathrm{P}_{\mathrm{E}}$ are much stronger and, consequently, more interesting. For example, light $\mathrm{P}_{\mathrm{E}}$ with $M_{\mathrm{P}_{\mathrm{E}}}<60 \mathrm{GeV}$ would be allowed only in models where $N_{\mathrm{TC}} \leq 12$, provided that $f_{\mathrm{P}_{\mathrm{E}}}<v / 3$. Heavier $\mathrm{P}_{\mathrm{E}}$, with masses in the range from $60 \mathrm{GeV}$ to $120 \mathrm{GeV}$, would be excluded for $f_{\mathrm{P}_{\mathrm{E}}} \lesssim v / 6$ and would be allowed only in models with $N_{\mathrm{TC}} \lesssim 10$ even if $f_{\mathrm{P}_{\mathrm{E}}}$ were as large as $v / 2$.

One way to obtain PNGBs with larger anomaly factors is to include technifermions in larger representations of $\mathrm{SU}(2)_{L}$. Manohar and Randall created [1] a one-family model with a weak isotriplet of left-handed techniquarks, Q, of hypercharge $Y=y$ and a weak isotriplet of left-handed technileptons, L, of hypercharge $Y=-3 y$; the right-handed technifermions are weak singlets. In the absence of isospin breaking, the technipion decay constant is $f_{\mathrm{Pa}^{\mathrm{a}}}=v / 4=61.5 \mathrm{GeV}$. There are four neutral PNGBs, with generators

$$
\begin{aligned}
& P^{1}=\frac{1}{6 \sqrt{2}}\left(3 \overline{\mathrm{L}} \gamma_{5} \mathrm{~L}-\overline{\mathrm{Q}} \gamma_{5} \mathrm{Q}\right) \quad P^{3}=\frac{1}{2 \sqrt{3}}\left(3 \overline{\mathrm{L}} \gamma_{5} \tau^{3} \mathrm{~L}-\overline{\mathrm{Q}} \gamma_{5} \tau^{3} \mathrm{Q}\right) \\
& P_{-}^{5}=\frac{1}{2 \sqrt{3}}\left(3 \overline{\mathrm{L}} \gamma_{5} \tau^{8} \mathrm{~L}-\overline{\mathrm{Q}} \gamma_{5} \tau^{8} \mathrm{Q}\right) \quad P_{+}^{5}=\frac{1}{2}\left(\overline{\mathrm{L}} \gamma_{5} \tau^{8} \mathrm{~L}+\overline{\mathrm{Q}} \gamma_{5} \tau^{8} \mathrm{Q}\right)
\end{aligned}
$$

where $\tau^{3}=\frac{1}{2} \operatorname{diag}(1,0,-1)$ and $\tau^{8}=\frac{1}{\sqrt{12}} \operatorname{diag}(1,-2,1)$. The corresponding anomaly factors are

$$
\begin{gathered}
\mathcal{A}_{\gamma \gamma}^{1}=6 \sqrt{2} y^{2} \approx 8.485 y^{2} \quad \mathcal{A}_{\mathrm{Z}^{0} \gamma}^{1}=6 \sqrt{2} y^{2} s_{W}^{2} \approx 1.948 y^{2} \quad \mathcal{A}_{\mathrm{Z}^{0} \mathrm{Z}^{0}}^{1}=6 \sqrt{2} y^{2} s_{W}^{4} \approx 0.449 y^{2} \\
\mathcal{A}_{\gamma \gamma}^{3}=4 \sqrt{3} y \approx 6.928 y \quad \mathcal{A}_{\mathrm{Z}^{0} \gamma}^{3}=\sqrt{3}\left(1-4 s_{W}^{2}\right) y \approx 0.139 y \quad \mathcal{A}_{\mathrm{Z}^{0} \mathrm{Z}^{0}}^{3}=2 \sqrt{3} s_{W}^{2}\left(1-2 s_{W}^{2}\right) y \approx 0.430 y \\
\mathcal{A}_{\gamma \gamma}^{5-}=\mathcal{A}_{\mathrm{Z}^{0} \gamma}^{5-}=\mathcal{A}_{\mathrm{Z}^{0} \mathrm{Z}^{0}}^{5-}=0 \\
\mathcal{A}_{\gamma \gamma}^{5+}=\frac{2}{\sqrt{3}} \approx 1.155 \quad \mathcal{A}_{\mathrm{Z}^{0} \gamma}^{5+}=\frac{1}{\sqrt{3}}\left(1-2 s_{W}^{2}\right) \approx 0.312 \quad \mathcal{A}_{\mathrm{Z}^{0} \mathrm{Z}^{0}}^{5+}=\frac{1}{\sqrt{3}}\left(1-2 s_{W}^{2}+2 s_{W}^{4}\right) \approx 0.373 .
\end{gathered}
$$

LEP provides no information on $\mathrm{P}_{-}^{5}$, since this PNGB has no coupling to the $\mathrm{Z}^{0}$, $\gamma$, or $\mathrm{f} \overline{\mathrm{f}}$ pairs. ${ }^{7}$ For the other scalars, combining Equation 6.6 and the results in Tables 1 and 2, we find upper bounds on the size of the technicolor group as a function of $M_{\mathrm{Pa}}$ and $y$. These limits are given in Table 4.

As an example of what these results reveal about particular models, suppose we are interested in a theory with $N_{\mathrm{TC}}=4$ and techniquark hypercharge $y \sim 1$. No matter how the $\mathrm{P}^{1}$ state decays, the LEP data imply that its mass must be greater than $120 \mathrm{GeV}$. The lower bound on the mass of the $\mathrm{P}^{3}$ state depends sensitively on its dominant decay mode: invisible decays would have been seen if $\mathrm{P}^{3}$ had a mass below $120 \mathrm{GeV}$; diphoton decays would have been seen if the $\mathrm{P}^{3}$ mass is below $160 \mathrm{GeV}$; a $\mathrm{P}^{3}$ decaying to $\mathrm{b} \overline{\mathrm{b}}$ is excuded unless its mass lies in the range $30 \mathrm{GeV}<M_{\mathrm{P}^{3}}<60 \mathrm{GeV}$. Finally, if the $\mathrm{P}_{+}^{5}$ leads to two-photon final states, its mass must be greater than about $125 \mathrm{GeV}$; if it decays to $\mathbb{E}$ states, its mass must be greater than about

\footnotetext{
${ }^{7}$ The $\mathrm{P}_{-}^{5}$ does not couple to a pair of neutral electroweak bosons since the anomaly factors vanish. Because the $\mathrm{P}_{-}^{5}$ and $\mathrm{P}_{+}^{5}$ are isospin two resonances, they do not couple to $\mathrm{f} \overline{\mathrm{f}}$. The $\mathrm{P}_{-}^{5}$ is not stable, however, since it can decay via $\mathrm{QCD}$ gluons, technigluons or Extended Technicolor gauge bosons.
} 
Table 5: Limits on the number of technicolors, $N_{\mathrm{TC}}$, and weak doublets of technifermions, $N_{D}$, for hadronically decaying PNGBs in TCSM [29, 30] models as a function of the upper bound on the PNGB mass, from Section 6.2. The superscripted labels indicate the data used to calculate the limits: $\dagger: \mathcal{A}_{\gamma \gamma}$ from Table $1 ; \ddagger$ : $\mathcal{A}_{\mathrm{Z}^{0} \gamma}$ from Table 1 .

\begin{tabular}{|c|c|c|}
\hline$M_{\pi_{\mathrm{T}}^{0 \prime}} \leq$ & \multicolumn{2}{|c|}{$N_{\mathrm{TC}} \sqrt{N_{D}} \leq$} \\
& $\pi_{\mathrm{T}}^{0 \prime} \rightarrow \mathrm{gg}$ & $\pi_{\mathrm{T}}^{0 \prime} \rightarrow \mathrm{b} \overline{\mathrm{b}}$ \\
\hline $30 \mathrm{GeV}$ & $28^{\ddagger}$ & $24^{\ddagger}$ \\
$60 \mathrm{GeV}$ & $67^{\ddagger}$ & $70^{\ddagger}$ \\
$80 \mathrm{GeV}$ & $283^{\ddagger}$ & $25^{\dagger}$ \\
$100 \mathrm{GeV}$ & - & $40^{\dagger}$ \\
$120 \mathrm{GeV}$ & - & $42^{\dagger}$ \\
$140 \mathrm{GeV}$ & - & $49^{\dagger}$ \\
$160 \mathrm{GeV}$ & - & $68^{\dagger}$ \\
\hline
\end{tabular}

$70 \mathrm{GeV}$. The bounds on the mass of $\mathrm{P}_{+}^{5}$ are insensitive to the value of hypercharge assumed; those for the other PNGB loosen as the hypercharge value decreases. The bounds become stricter if a larger technicolor group is chosen.

\subsection{Low-scale Technicolor Models}

Many modern technicolor models feature a "walking" technicolor coupling to eliminate large flavor-changing neutral currents $[19,31]$ and separate topcolor interactions $[32,33]$ to provide the large top quark mass. Both innovations tend to require the presence of a large number $N_{D}$ of weak doublets ${ }^{8}$ of technifermions. For a given technicolor gauge group $\mathrm{SU}\left(N_{\mathrm{TC}}\right)$, the number of doublets required to make the gauge coupling $g_{\mathrm{TC}}$ run slowly at scales above the characteristic technicolor scale, $\Lambda_{\mathrm{TC}}$, while remaining asymptotically free can be estimated from the one-loop beta function:

$$
\beta_{\mathrm{TC}}=-\frac{g_{\mathrm{TC}}^{3}}{16 \pi^{2}}\left(\frac{11}{3} N_{\mathrm{TC}}-\frac{4}{3} N_{D}\right)+\cdots
$$

In the models of refs. [35, 36, 37], for example, $N_{D} \approx 10$. Likewise, topcolor-assisted technicolor models appear to need many doublets of technifermions to accommodate the masses of the light fermions, the mixing between light and heavy fermions, and the dynamical breaking of topcolor [29, 35]. As mentioned in Section 2, a large number of doublets implies a small technipion decay constants, $f_{\mathrm{Pa}}=v / \sqrt{N_{D}}$.

As an example of a low-scale technicolor theory, we analyze Lane's Technicolor Straw Man Model (TCSM) $[29,30]$. We assume that the lightest technifermion doublet, composed of technileptons $\mathrm{T}_{\mathrm{U}}$ and $\mathrm{T}_{\mathrm{D}}$ with electric charges $Q_{U}$ and $Q_{D}$ respectively, can be considered in isolation. Following Lane, we take $Q_{U}=4 / 3$ and $Q_{D}=1 / 3$, and we assume that there are two, nearly degenerate neutral mass eigenstates, whose generators are given by

$$
P_{\pi_{\mathrm{T}}^{0}}=\frac{1}{2}\left(\overline{\mathrm{T}}_{\mathrm{U}} \gamma_{5} \mathrm{~T}_{\mathrm{U}}-\overline{\mathrm{T}}_{\mathrm{D}} \gamma_{5} \mathrm{~T}_{\mathrm{D}}\right) \quad P_{\pi_{\mathrm{T}}^{0 \prime}}=\frac{1}{2}\left(\overline{\mathrm{T}}_{\mathrm{U}} \gamma_{5} \mathrm{~T}_{\mathrm{U}}+\overline{\mathrm{T}}_{\mathrm{D}} \gamma_{5} \mathrm{~T}_{\mathrm{D}}\right) .
$$

\footnotetext{
${ }^{8}$ While estimates of the $S$ and $T$ parameters in technicolor theories assumed to have QCD-like dynamics seem to suggest that the number of technifermion doublets must be small, such estimates cease to apply if the technicolor coupling remains strong out to the Extended Technicolor scale as in walking models [34].
} 
Table 6: Limits on the number of technicolors $N_{\mathrm{TC}}$ in the walking technicolor model ofLane and Ramana [4, 40] as a function of the upper bound on the $\mathrm{P}_{\mathrm{L}}^{3}$ mass, from Section 6.2. Note that $f_{\mathrm{P}_{\mathrm{L}}^{3}}=41 \mathrm{GeV}$. The superscripted labels indicate the data used to calculate the limits: $\dagger: \mathcal{A}_{\gamma \gamma}$ from Table $1 ; \ddagger: \mathcal{A}_{\mathrm{Z}^{0} \gamma}$ from Table $1 ; \#: \mathcal{A}_{\gamma \gamma}$ from Table 2 .

\begin{tabular}{|c|c|c|}
\hline$M_{\mathrm{P}_{\mathrm{L}}^{3}} \leq$ & \multicolumn{2}{|c|}{$N_{\mathrm{TC}} \leq$} \\
& $\mathrm{P}_{\mathrm{L}}^{3} \rightarrow \gamma \gamma$ & $\mathrm{P}_{\mathrm{L}}^{3} \rightarrow \mathrm{bb}$ \\
\hline $30 \mathrm{GeV}$ & $3.5^{\#}$ & $14.7^{\ddagger}$ \\
$60 \mathrm{GeV}$ & $3.3^{\#}$ & $42.6^{\ddagger}$ \\
$80 \mathrm{GeV}$ & $1.4^{\dagger}$ & $3.3^{\dagger}$ \\
$100 \mathrm{GeV}$ & $1.6^{\dagger}$ & $5.1^{\dagger}$ \\
$120 \mathrm{GeV}$ & $1.9^{\dagger}$ & $5.4^{\dagger}$ \\
$140 \mathrm{GeV}$ & $3.5^{\dagger}$ & $6.2^{\dagger}$ \\
$160 \mathrm{GeV}$ & $5.1^{\dagger}$ & $8.7^{\dagger}$ \\
\hline
\end{tabular}

We can then calculate the relevant anomaly factors

$$
\begin{array}{lll}
\mathcal{A}_{\gamma \gamma}^{\pi_{\mathrm{T}}^{0}}=\frac{5}{6} \approx 0.833 & \mathcal{A}_{\mathrm{Z}^{0} \gamma}^{\pi_{\mathrm{T}}^{0}}=\frac{5}{24}\left(1-4 s_{W}^{2}\right) \approx 0.017 & \mathcal{A}_{\mathrm{Z}^{0} \mathrm{Z}^{0}}^{\pi_{\mathrm{T}}^{0}}=\frac{5}{12} s_{W}^{2}\left(1-2 s_{W}^{2}\right) \approx 0.225 \\
\mathcal{A}_{\gamma \gamma}^{\pi_{\mathrm{T}}^{0 \prime}}=\frac{17}{18} \approx 0.944 & \mathcal{A}_{\mathrm{Z}^{0} \gamma}^{\pi_{\mathrm{T}}^{0 \prime}}=\frac{17}{18} s_{W}^{2}-\frac{1}{8} \approx 0.092 & \mathcal{A}_{\mathrm{Z}^{0} \mathrm{Z}^{0}}^{\pi_{\mathrm{T}}^{0 \prime}}=\frac{1}{8}-\frac{1}{4} s_{W}^{2}+\frac{17}{18} s_{W}^{4} \approx 0.117 .
\end{array}
$$

We further assume [30] that these PNGBs decay to jets, with $\pi_{\mathrm{T}}^{0} \rightarrow \mathrm{b} \overline{\mathrm{b}}$ and $\pi_{\mathrm{T}}^{0 \prime} \rightarrow \mathrm{gg}, \mathrm{b} \overline{\mathrm{b}}$ dominating.

From the limits on $N_{\mathrm{TC}} \mathcal{A}_{\mathrm{Z}^{0} \mathrm{Z}^{0}}$ obtained in Section 3.2.1 for $\mathrm{Z}^{0} \rightarrow \mathrm{Z}^{*} \mathrm{P}^{\mathrm{a}}$ with $\mathrm{P}^{\mathrm{a}} \rightarrow j j$ and $\mathrm{Z}^{*} \rightarrow \nu \bar{\nu}$, we can use the value of $\mathcal{A}_{\mathrm{Z}^{0} \mathrm{Z}^{0}}^{\pi_{0}^{0}}$ above, to find $N_{\mathrm{TC}} \leq 225 / \sqrt{N_{D}}$ for $M_{\pi_{\mathrm{T}}^{0}} \leq 30 \mathrm{GeV}$. Unfortunately, this does not provide interesting limits even for this small $M_{\pi_{\mathrm{T}}^{0}}$.

More useful is the bound that can be obtained by combining the value of $\mathcal{A}_{\mathrm{Z}^{0} \gamma}^{\pi_{\mathrm{T}}^{0 \prime}}$ and $\mathcal{A}_{\gamma \gamma}^{\pi_{\mathrm{T}}^{0 \prime}}$ above with the limits on $N_{\mathrm{TC}} \mathcal{A}_{\mathrm{Z}^{0} \gamma}$ and $N_{\mathrm{TC}} \mathcal{A}_{\gamma \gamma}$ obtained for hadronic $\mathrm{P}^{\mathrm{a}}$ decays in Tables 1 and 2. For the decay modes $\pi_{\mathrm{T}}^{0 \prime} \rightarrow$ gg or b $\bar{b}$ we find upper bounds on $N_{\mathrm{TC}} \sqrt{N_{D}}$ as a function of $M_{\pi_{\mathrm{T}}^{0 \prime}}$, as summarized in Table 5 .

To clarify the meaning of these bounds, we now consider the case where $M_{\pi_{\mathrm{T}}^{0 \prime}} \leq 30 \mathrm{GeV}$ and the $\pi_{\mathrm{T}}^{0 \prime}$ decays primarily to b quarks; in this case the limit $N_{\mathrm{TC}} \sqrt{N_{D}} \leq 24$ applies. As a result, for $N_{\mathrm{TC}}=(4,6,8,10,12)$ the largest number of electroweak doublets of technifermions allowed by the LEP data is, respectively, $N_{D}=(36,16,9,5,4)$. The results are very similar if the two-gluon decays of the PNGB dominate instead.

How do these results accord with the requirements of walking technicolor? Based on the one-loop technicolor beta function, $\beta_{\mathrm{TC}}$ (Equation 6.7), a slowly running $g_{\mathrm{TC}}$ requires the presence of about $11 N_{\mathrm{TC}} / 4$ weak doublets of technifermions. Then according to the LEP data, walking technicolor and a very light $\pi_{\mathrm{T}}^{0 \prime}$ $\left(M_{\pi_{\mathrm{T}}^{0 \prime}}<30 \mathrm{GeV}\right)$ can coexist only in models with $N_{\mathrm{TC}}=4$ or 6 . A similar analysis of cases with heavier $\pi_{\mathrm{T}}^{0 \prime}$ shows that the size of the technicolor group is restricted to $N_{\mathrm{TC}} \leq 6$ if $M_{\pi_{\mathrm{T}}^{0 \prime}}=80 \mathrm{GeV}$, loosening to $N_{\mathrm{TC}} \leq 12$ for a $160 \mathrm{GeV} \pi_{\mathrm{T}}^{0 \prime}$. The results are similar if the 2-loop $\beta_{\mathrm{TC}}$ function is used ${ }^{9}$, even for a moderately strong technicolor coupling $g_{\mathrm{TC}}^{2} / 4 \pi \sim 1$.

As a second example, we mention what our results imply for the walking technicolor model of Lane and Ramana [40] whose LEP II and NLC phenomenology was studied by Lubicz and Santorelli [4]. To make

\footnotetext{
${ }^{9}$ The two loop correction to $\beta_{\mathrm{TC}}$ includes the additional term $[38,39]$

$$
-\frac{g_{\mathrm{TC}}^{5}}{\left(16 \pi^{2}\right)^{2}}\left(\frac{34}{3} N_{\mathrm{TC}}^{2}-\frac{26}{3} N_{\mathrm{TC}} N_{D}+2 \frac{N_{D}}{N_{\mathrm{TC}}}\right) .
$$
}


contact with their analysis, we follow them in taking $N_{D}=9$ : one color-triplet of techniquarks $\left(N_{Q}=1\right)$ and six color-singlets of technileptons $\left(N_{L}=6\right)$. Of the several neutral PNGBs in this model, the one whose relatively large anomaly factors and small decay constant makes it easiest to produce is

$$
P_{\mathrm{L}}^{3}=\frac{1}{2}\left(\overline{\mathrm{N}}_{l} \gamma_{5} \mathrm{~N}_{l}-\overline{\mathrm{E}}_{l} \gamma_{5} \mathrm{E}_{l}\right)
$$

where the subscript implies a sum over all $N_{L}$ technilepton doublets. This PNGB has a decay constant $f_{\mathrm{P}_{\mathrm{L}}^{3}}=41 \mathrm{GeV}$, and anomaly factors (in our normalization),

$$
\mathcal{A}_{\gamma \gamma}^{\mathrm{P}_{L}^{3}}=\frac{\sqrt{N_{L}}}{2} \approx 1.225 \quad \mathcal{A}_{\mathrm{Z}^{0} \gamma}^{\mathrm{P}_{\mathrm{L}}^{3}}=\frac{\sqrt{N_{L}}}{8}\left(1-4 s_{W}^{2}\right) \approx 0.024 \quad \mathcal{A}_{\mathrm{Z}^{0} \mathrm{Z}^{0}}^{\mathrm{P}_{\mathrm{L}}^{3}}=\frac{\sqrt{N_{L}}}{4} s_{W}^{2}\left(1-2 s_{W}^{2}\right) \approx 0.076,
$$

where the numerical factors are for $N_{L}=6$. This PNGB is expected to have a mass in the range $100-350 \mathrm{GeV}$ [4]. Depending on the value of the ETC coupling between the PNGB and fermions, the dominant decay of this PNGB may be into a photon pair or $\mathrm{b} \overline{\mathrm{b}}$. In Table 6 , we show the upper bound on the size of the technicolor group as a function of PNGB mass implied by the results in Tables 1 and 2. Apparently, if the two-photon decays dominate, the PNGB must have a mass in excess of $160 \mathrm{GeV}$; if the $\mathrm{b} \overline{\mathrm{b}}$ decay is preferred, the mass range $80 \mathrm{GeV} \leq M_{\mathrm{Pa}} \leq 120 \mathrm{GeV}$ is excluded.

\section{Conclusions}

Using published analyses of data from LEP I and LEP II, we have derived improved limits on the anomalous PNGB couplings to $\mathrm{Z}^{0} \gamma$ and the first limits on couplings to $\mathrm{Z}^{0} \mathrm{Z}^{0}$ and $\gamma \gamma$. For models in which the PNGBs decay to photons or hadrons, the bounds on $N_{\mathrm{TC}} \mathcal{A}_{\mathrm{Z}^{0} \gamma}$ are a factor of 2-3 stronger than those previously reported [3]; for PNGBs manifesting as missing energy, the bounds are of similar strength but extend over a larger mass range. As a result, it is possible to set useful constraints on the existence of light PNGBs in non-minimal technicolor models that have large anomalous couplings of the PNGBs to $Z^{0} \gamma, Z^{0} Z^{0}$, and $\gamma \gamma$ and small technipion decay constants. For example, the data are sensitive to light $\pi_{\mathrm{T}}^{0 \prime}$ in models of low-scale technicolor which typically include of order 10 weak doublets of technifermions or in models with weak isotriplet technifermions.

Substantial further improvements of the limits for light $\mathrm{P}^{\mathrm{a}} M_{\mathrm{P}^{\mathrm{a}}}<M_{\mathrm{Z}^{0}}$, will require further data collection at the $\mathrm{Z}^{0}$ pole. Operation on the $\mathrm{Z}^{0}$ resonance in the GigaZ mode of TESLA [41], for example, should produce more than $10^{9} \mathrm{Z}^{0}$ events per year of operation. This would generate one thousand times more data per year of running than was collected by any one of the LEP experiments. Assuming that the limits derived by the LEP collaborations are constrained by statistics, this quantity of data should allow improvements in the cross section limits by a factor of 30 , which would lead to an improvement of at least a factor of five in most of our limits on both $N_{\mathrm{TC}} \mathcal{A}_{\mathrm{Z}^{0} \gamma}$ and $N_{\mathrm{TC}} \mathcal{A}_{\mathrm{Z}^{0} \mathrm{Z}^{0}}$.

The search for heavier $\mathrm{P}^{\mathrm{a}}$ can be extended in several ways. In the short term, analysis of the complete LEP II data sample should increase the reach of each experiment. Combining the results from different experiments could also give some improvement in the bounds. In the long term, a high energy high luminosity $\mathrm{e}^{+} \mathrm{e}^{-}$ collider will be able to search for PNGBs with higher masses, larger decay constants, and smaller couplings [4].

\section{Acknowledgments}

The authors thank R.S. Chivukula for comments on the manuscript. E.H.S. acknowledges the support of the NSF Professional Opportunities for Women in Research and Education (POWRE) program, and the 
hospitality of the Harvard University Physics Department. This work was supported in part by the National Science Foundation under grant PHY-0074274, and by the Department of Energy under grant DE-FG0291ER40676.

\section{References}

[1] A. Manohar and L. Randall, Phys. Lett. B 246, 537 (1990).

[2] L. Randall and E. H. Simmons, Nucl. Phys. B 380, 3 (1992).

[3] G. Rupak and E. H. Simmons, Phys. Lett. B 362, 155 (1995), hep-ph/9507438.

[4] V. Lubicz and P. Santorelli, Nucl. Phys. B 460, 3 (1996), hep-ph/9505336.

[5] R. Casalbuoni et al., Nucl. Phys. B 555, 3 (1999), hep-ph/9809523.

[6] M. Acciarri et al. (L3), Phys. Lett. B345, 609 (1995), CERN-PPE/94-186.

[7] P. Abreu et al. (DELPHI), Z. Phys. C74, 577 (1997), CERN-PPE/96-003.

[8] M. Acciarri et al. (L3), Phys. Lett. B 388, 409 (1996), CERN-PPE/96-050.

[9] G. Alexander et al. (OPAL), Z. Phys. C71, 1 (1996), CERN-PPE/95-193.

[10] G. Alexander et al. (OPAL), Phys. Lett. B 377, 273 (1996), CERN-PPE/96-019.

[11] G. Abbiendi et al., Phys. Lett. B 465, 303 (1999), hep-ex/9907064.

[12] P. Abreu et al., Phys. Lett. B 458, 431 (1999), CERN-EP-99-058.

[13] M. Acciarri et al., Phys. Lett. B 489, 102 (2000), hep-ex/0008023.

[14] P. Abreu et al., Eur. Phys. J. C17, 53 (2000), CERN-EP-2000-021.

[15] R. Barate et al., Phys. Lett. B 466, 50 (1999), CERN-EP-99-125.

[16] M. Acciarri et al., Phys. Lett. B 489, 115 (2000), hep-ex/0008025, CERN-EP-2000-103.

[17] S. Dimopoulos, S. Raby, and G. L. Kane, Nucl. Phys. B 182, 77 (1981).

[18] J. Ellis, M. K. Gaillard, D. V. Nanopoulos, and P. Sikivie, Nucl. Phys. B 182, 529 (1981).

[19] B. Holdom, Phys. Rev. D 24, 157 (1981).

[20] D. Groom et al. (Particle Data Group), Eur. Phys. J. C15, 1 (2000), URL http://pdg. lbl.gov.

[21] R. Akers et al. (OPAL), Z. Phys. C65, 47 (1995), CERN-PPE/94-105.

[22] M. Acciarri et al. (L3), Phys. Lett. B 346, 190 (1995), CERN-PPE/94-216.

[23] T. Appelquist and J. Terning, Phys. Lett. B 315, 139 (1993), hep-ph/9305258.

[24] F. Berends and R. Kleiss, Nucl. Phys. B 260, 32 (1985).

[25] J. F. Gunion, H. E. Haber, G. Kane, and S. Dawson, The Higgs Hunter's Guide (Addison-Wesley Publishing Company, Reading, MA, 1990). 
[26] B. Ioffe and V. Khoze, Sov. J. Nucl. Phys. 9, 50 (1978).

[27] B. E. Lee, C. Quigg, and H. B. Thacker, Phys. Rev. D 16, 1519 (1977).

[28] E. Farhi and L. Susskind, Phys. Rep. 74, 277 (1981).

[29] K. Lane, Phys. Rev. D 60, 075007 (1999), hep-ph/9903369.

[30] K. Lane, Technihadron production and decay rates in the Technicolor Straw Man Model (1999), companion to [29], hep-ph/9903372.

[31] B. Holdom, Phys. Lett. B 150, 301 (1985).

[32] C. T. Hill, Phys. Lett. B 266, 419 (1991).

[33] C. T. Hill, Phys. Lett. B 345, 483 (1995), hep-ph/9411426.

[34] K. Lane, in International Conference on High Energy Physics, 27th, edited by P. J. Bussey and I. G. Knowles (IOP, 1994), pp. 543-548, hep-ph/9409304.

[35] K. Lane and E. Eichten, Phys. Lett. B352, 382 (1995), hep-ph/9503433.

[36] K. Lane, Phys. Rev. D 54, 2204 (1996), htp-ph/9602221.

[37] K. Lane, Phys. Lett. B 433, 96 (1998), hep-ph/9805254.

[38] W. Caswell, Phys. Rev. Lett. 33, 244 (1974).

[39] D. R. T. Jones, Nucl. Phys. B 75, 531 (1974).

[40] K. Lane and M. V. Ramana, Phys. Rev. D D44, 2678 (1991).

[41] J. Erler, S. Heinemeyer, W. Hollik, G. Weiglein, and P. Zerwas, Phys. Lett. B 486, 125 (2000), hep-ph/0005024. 